\title{
Oligosaccharides
}

\section{Advances in Stereoselective 1,2-cis Glycosylation using C-2 Auxiliaries}

Rens A. Mensink and Thomas J. Boltje*[a]

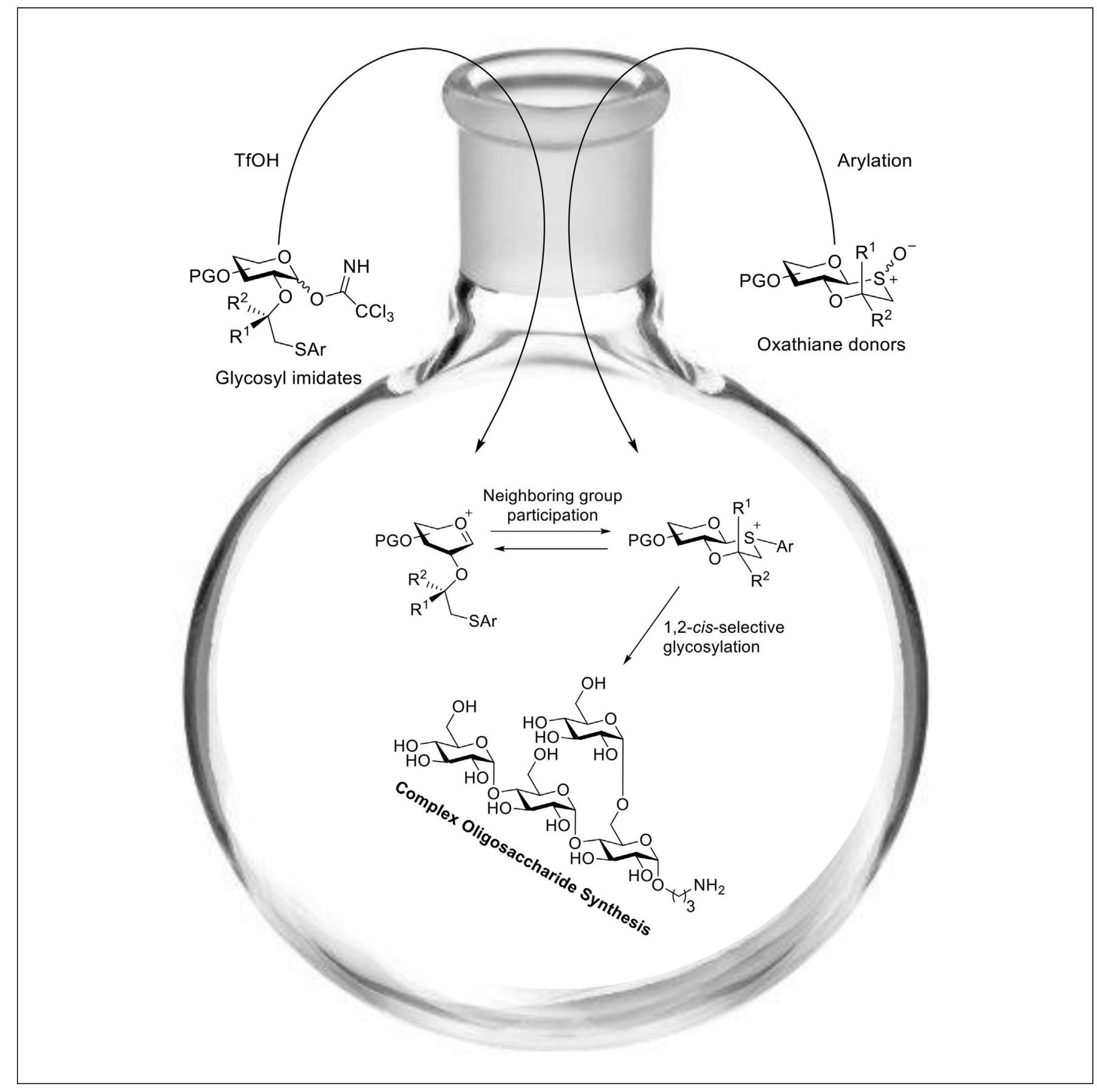


Abstract: The control of stereoselectivity in a glycosylation reaction remains one of the most challenging aspects of oligosaccharide synthesis. Especially the synthesis of 1,2-cis-glycosides is challenging and generally applicable methodology to prepare this linkage is needed to standardize oligosaccharide synthesis. This review highlights the recent development of an elegant strategy employing a C-2 auxiliary to control the anomeric stereoselectivity in glycosylations. The various auxiliaries developed to date, their compatibility with protecting groups and monosaccharide types as well as mechanistic aspects are summarized. Furthermore, the application, advantages and limitations of C-2 auxiliaries in oligosaccharide synthesis are discussed.

\section{Introduction}

The three major classes of macromolecules in biological systems comprise DNA, proteins and carbohydrates (also called glycans or saccharides). ${ }^{[1]}$ Information encoded in DNA is transcribed and translated to direct protein synthesis. Hence, with the elucidation of the human genome came the expectation that all biological processes could be understood. However, our genetic blueprint proved insufficient to account for the origin of all biological processes. Other information-rich molecules that are not under direct genetic control must therefore play a major role. ${ }^{[2]}$ Carbohydrates are a very important class of molecules in this respect and hence there is a growing appreciation that glycosylation dramatically increases protein complexity and function. ${ }^{[3]}$

Similar to genomics for DNA and proteomics for proteins, "glycomics" is the study that seeks to identify and understand the structure and function of specific carbohydrate patterns in biological processes and the launch of the human glycome project is imminent. ${ }^{[3]}$ A major obstacle in relating carbohydrate structure to function is the lack of pure and structurally well-defined carbohydrates and glycoconjugates. Because these compounds are difficult to isolate from natural sources, well-defined oligosaccharides can often only be obtained by chemical or enzymatic synthesis. ${ }^{[4]}$

\section{Oligosaccharide and glycosidic linkage structure}

Oligosaccharides are complex structures since they are often highly branched and the monomeric constituents (monosaccharides) are connected by glycosidic bonds that exist as either equatorial or axial diastereoisomers, called anomers. The anomeric stereochemistry is usually defined relative to the C-2 substituent $(\mathrm{X})$, which can be axial (manno-type) or equatorial (gluco-type), as 1,2-cis or 1,2-trans, giving rise to four possibilities (Figure $1 \mathrm{~A}$ ). The anomeric configuration can also be defined as $\alpha$-(1,2-cis-D-glucotype and 1,2-trans-D-mannotype) or $\beta$-anomers (1,2-cis-D-mannotype and 1,2-trans-D-glucotype). In addition, the C-2 substituent is most frequently an alcohol,

[a] R. A. Mensink, Dr. T. J. Boltje

Institute for Molecules and Materials (IMM)

Radboud University Nijmegen

Heyendaalseweg 135, 6525 AJ, Nijmegen (The Netherlands)

E-mail: t.boltje@science.ru.nl

(D) The ORCID identification number(s) for the author(s) of this article can be found under https://doi.org/10.1002/chem.201700908.

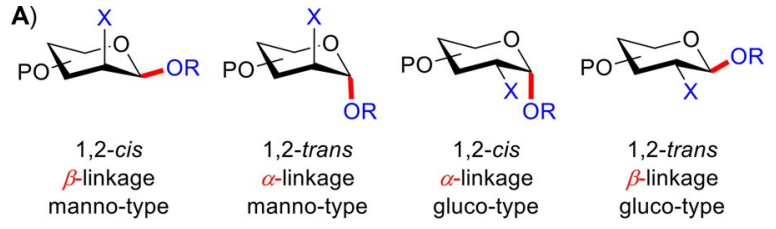

B)

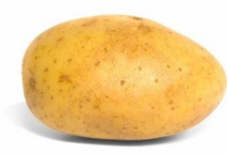

Potato

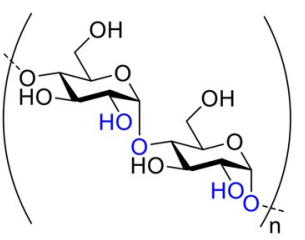

Amylose: Glc- $\alpha-1,4 \mathrm{Glc}$, Sweet taste
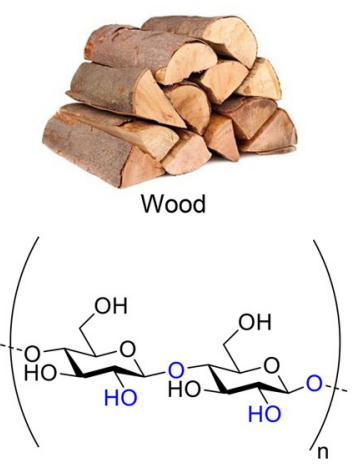

Cellulose: GIc- $\beta-1,4 \mathrm{Glc}$ Structural component of wood
Figure 1. Structures of anomeric linkages. A) Four different kinds of linkages found in nature, B) The anomeric configuration has a profound influence on the molecular structure, properties and biological function. For example, anomeric diastereoisomers amylose and cellulose have a different biological role as the first is a food source and the latter a structural component of wood.

amine or hydrogen. The structural differences in the C-2 substituent and its relative orientation with respect to the anomeric substituent may seem subtle, but can have major implications for the biological function. For example, amylose and cellulose are anomeric diastereoisomers of 1,4-linked glucose polymers, and while amylose is a food source and can be enzymatically converted into glucose, cellulose on the other hand forms very strong fibers and is a structural component of wood (Figure 1 B).

With the importance of anomeric selectivity in mind, the main challenge in carbohydrate synthesis is the regioselective and stereoselective introduction of glycosidic bonds. ${ }^{[4,5]}$ Similar to other stereoselective reactions, the induction of diastereoselectivity during a glycosylation event can be achieved by the use of a chiral catalyst, neighboring group participation (NGP) and creating facial selectivity using remote chiral centers. ${ }^{[4,5]}$ Since most of these methods rely on the relative stereochemistry of neighboring and remote stereocenters, methods that are effective for glucose are not necessarily applicable to galactose or mannose and vice versa. A generally applicable glycosyla- 
A)

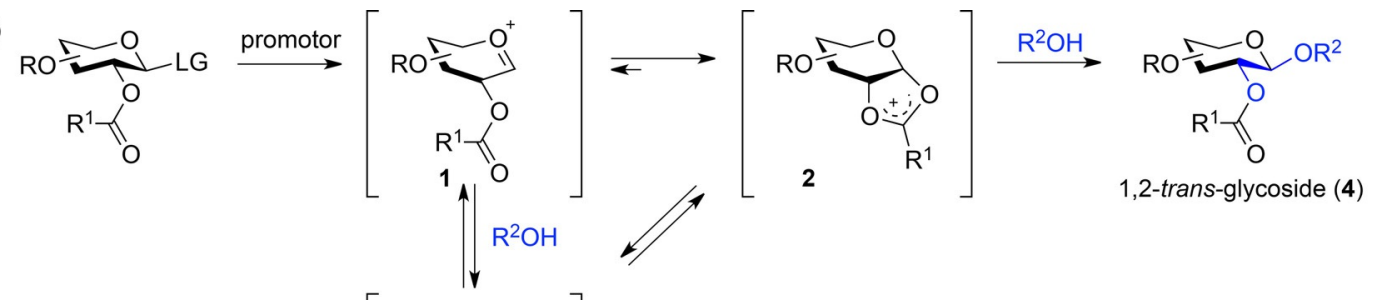

B)

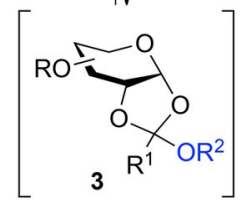

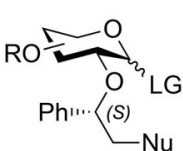

5

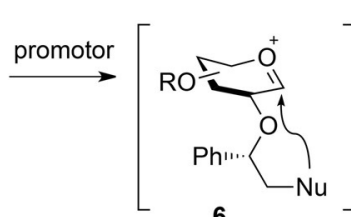
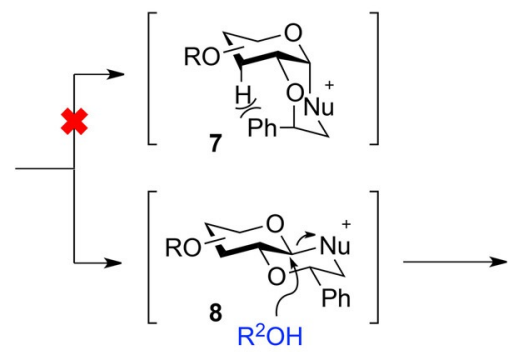

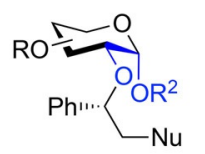

1,2-cis-glycoside (9)

C)

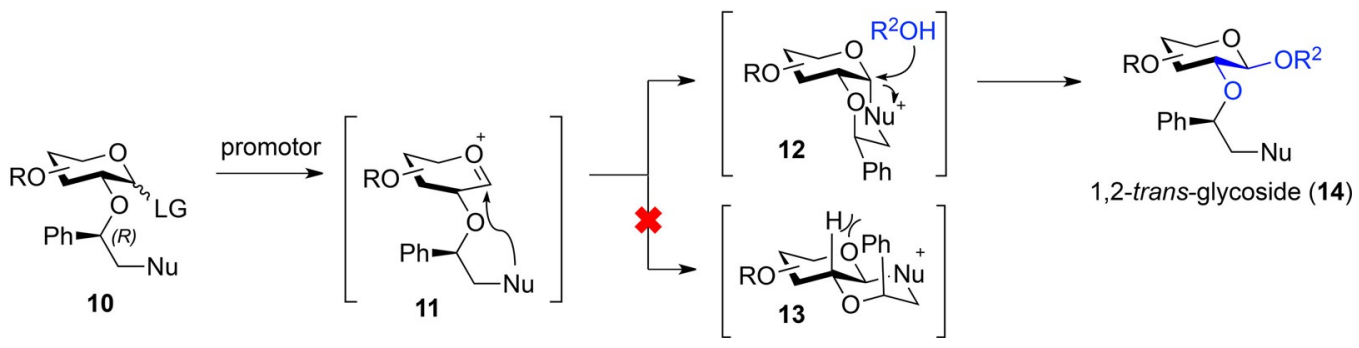

Scheme 1. Chiral auxiliaries designed to form cis- or trans-decalin intermediates to afford 1,2-trans or 1,2-cis-glycosides, respectively. A) C-2 acyl participation reliably affords 1,2-trans glycosides (4), B) A chiral S-configured auxiliary affords 1,2-cis glycosides (9) via a trans-configured sulfonium ion (8) C) A chiral $R$ configured auxiliary affords 1,2-trans glycosides (14) via a cis-configured sulfonium ion (12).

tion methodology is needed to make oligosaccharide synthesis routine work, similar to peptide and oligonucleotide synthesis. $^{[6]}$

General methods to prepare 1,2-trans glycosides are available and exploit neighboring group participation of a C-2-acyl group (Scheme $1 \mathrm{~A}){ }^{[5]}$ When a glycosyl donor carrying an anomeric leaving group is activated to form intermediate oxocarbenium ion 1, this intermediate is rapidly trapped by the neighboring group to form intermediate 2 , which can only be formed as a 1,2-cis fused ring system. A glycosyl acceptor $\left(\mathrm{R}^{2} \mathrm{OH}\right)$ can only attack 2 at the anomeric center from the $\beta$ face leading to the stereoselective formation of 1,2-trans glycoside 4. The glycosyl acceptor can also add directly to the dioxolenium ion (2) leading to the formation of orthoester 3 . The orthoester is not stable under acidic conditions and will rearrange to the desired 1,2-trans glycoside. Numerous 2-O-acyl protecting groups have been developed to reduce orthoester formation and/or incorporate a latent nucleophile to enable selective removal in the presence of other esters. For example, the bulky pivaloyl ester (trimethyl acetyl ester) can be used to suppress orthoester formation due to steric shielding of the dioxolenium ion. ${ }^{[7]}$

In general, the use of a 2-O-acyl functionality to synthesize 1,2-trans glycosides is very reliable, highly stereoselective and in the case of gluco-type donors, $\beta$-linked products will be ob-
Thomas Boltje studied organic chemistry at the university of Leiden, The Netherlands. He then moved to the Complex Carbohydrate Research Centre (CCRC, Georgia, USA) for his $\mathrm{PhD}$ studies on stereoselective glycosylation using chiral auxiliaries under the supervision of Prof. Dr. G.J. Boons. After a short postdoctoral period supported by a NWO Rubicon grant he became assistant professor in Nijmegen, The Netherlands, focusing on the chemistry and (chemical) biology of carbohydrates.

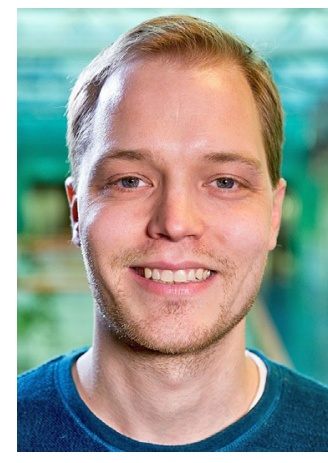

Rens Mensink obtained his MSc degree in synthetic organic chemistry in 2013 at the Radboud University in Nijmegen, The Netherlands. $\mathrm{He}$ is currently finishing his doctoral studies under the supervision of Dr. Thomas Boltje. His research is focused on stereoselective 1,2cis-glycosylations using neighboring group participation.

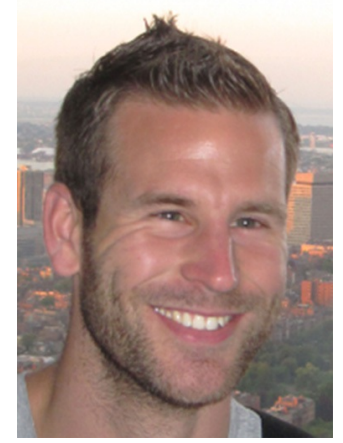


tained, whereas manno-type donors will give $\alpha$-linked products. Furthermore, this strategy is also applicable to the synthesis of 2-deoxy-2-amino-glycosides using amine-protecting groups that can perform NGP. However, care needs to be taken when using an $\mathrm{N}$-acetyl group for NGP as the corresponding glycosyl oxazoline is a commonly formed byproduct, but can be avoided by using Lewis acid catalysis. ${ }^{[8]}$ Alternatively, other amine protecting groups that can perform NGP such as phthalimido, tetrachlorophthalimido, trichloroacetyl, allyloxycarbonyl, and 2,2,2-trichloroethyloxycarbonyl can be used. ${ }^{[9]}$

In contrast, the introduction of 1,2-cis glycosidic linkages is much more challenging and requires glycosyl donors having a non-assisting functionality at $\mathrm{C}-2 .{ }^{[6]}$ In general, these glycosylations are less selective and require optimization to achieve acceptable anomeric ratios. Recent advances in anomeric control have led to new methodologies to synthesize 1,2-cis linkages of monosaccharides such as glucose, galactose, mannose, ${ }^{[10]}$ glucosamine, ${ }^{[11]}$ galactosamine, ${ }^{[12]}$ and furanose sugars. ${ }^{[13]} \mathrm{Al}-$ though excellent, these methods are stereoselective via different mechanisms ranging from solvent effects, ${ }^{[14]}$ directed aglycon delivery, ${ }^{[15]}$ steric shielding, ${ }^{[12]} \mathrm{S}_{\mathrm{N}}$ 2-like displacement of a quasi-stable intermediate, ${ }^{[10 a]}$ stabilization of an oxocarbenium ion conformation ${ }^{[13,16]}$ and post-glycosylation anomerization. ${ }^{[6,17]}$ Out of these methods, the directed aglycon delivery methodology, using a tether between the glycosyl donor and glycosyl acceptor to control anomeric selectivity is applicable to both gluco- and manno-type sugars. ${ }^{[15 b, c]}$ However, the installation of a tether is additional work and the glycosylation yields can be moderate. This methodology therefore still lacks the applicability and reliability of NGP to prepare 1,2-cis glycosides.

Herein, we review the development of a methodology that employs a C-2 chiral auxiliary to achieve 1,2-cis glycosylation. Similar to the use of NGP to prepare 1,2-trans glycosides, this methodology has the potential to become a generally applicable method for 1,2-cis glycoside synthesis. Combined, these complementary methods would enable the stereoselective synthesis of the most frequently found linkage types in nature and thereby advance the glycomics effort. ${ }^{[6]}$

\section{Chiral Auxiliaries for Glucose and Galactose}

The overall approach to achieve 1,2-cis stereoselectivity using $\mathrm{C}-2$ chiral auxiliaries is depicted in Scheme $1 \mathrm{~B}^{[18]}$ The chiral auxiliary is designed to trap the oxocarbenium ion from the $\beta$ face thereby blocking it and displacement of this intermediate should then result in the formation of 1,2-cis-glycosides. To achieve this, a nucleophilic group is situated to form a six membered ring system upon participation, which either results in a cis-(7) or trans-decalin (8) system. It was envisioned that a chiral substituent could be used to favor the formation of the cis or trans-decalin system leading to 1,2-trans or 1,2-cis glycosides, respectively. In case of an S-configured auxiliary, formation of cis-decaling intermediate 7 results in axial placement of the chiral substituent which in turn leads to an unfavorable 1,4-diaxial steric interaction. In contrast, formation of trans-decalin intermediate $\mathbf{8}$ leads to a more favorable equatorial substi- tution and is hence preferred. In addition, trans-decalin system 8 will have less gauche interactions than cis-decalin system 7. Therefore, the S-configuration is expected to favor 1,2-cis-glycoside formation by selectively forming trans-decalin intermediate 8. Similarly, the $R$-configured auxiliary was expected to favor 1,2-trans glycoside formation, since the cis-decalin intermediate (12) leads to equatorial placement of the phenyl substituent and prevents the 1,3-diaxial steric interaction that the trans-decalin (13) experiences (Scheme 1C).

\section{First and second generation chiral auxiliaries}

The first generation of chiral auxiliaries employed an (S)-ethoxycarbonylbenzyl moiety containing an ethyl ester as the nucleophilic head group since esters are proven to perform NGP.

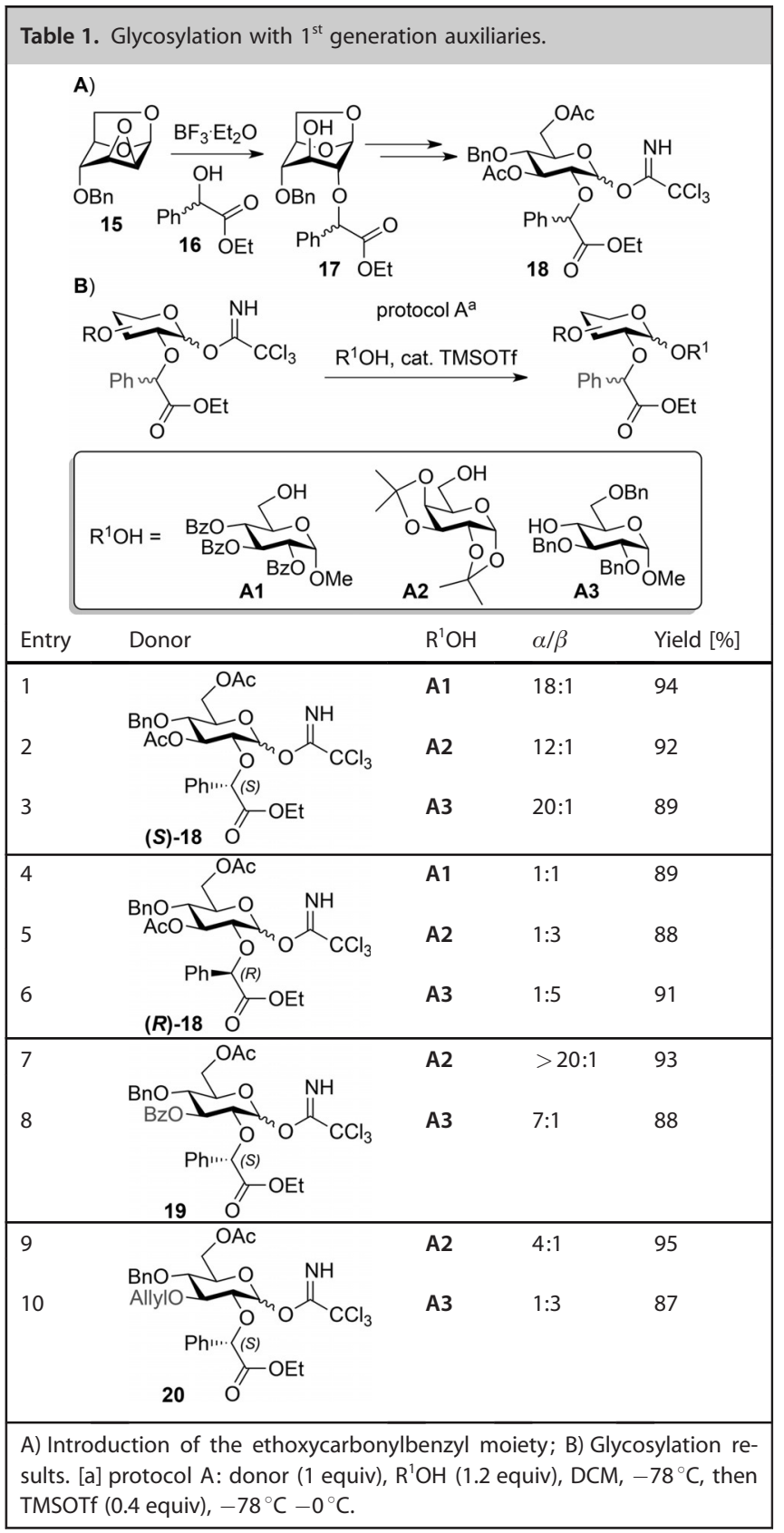




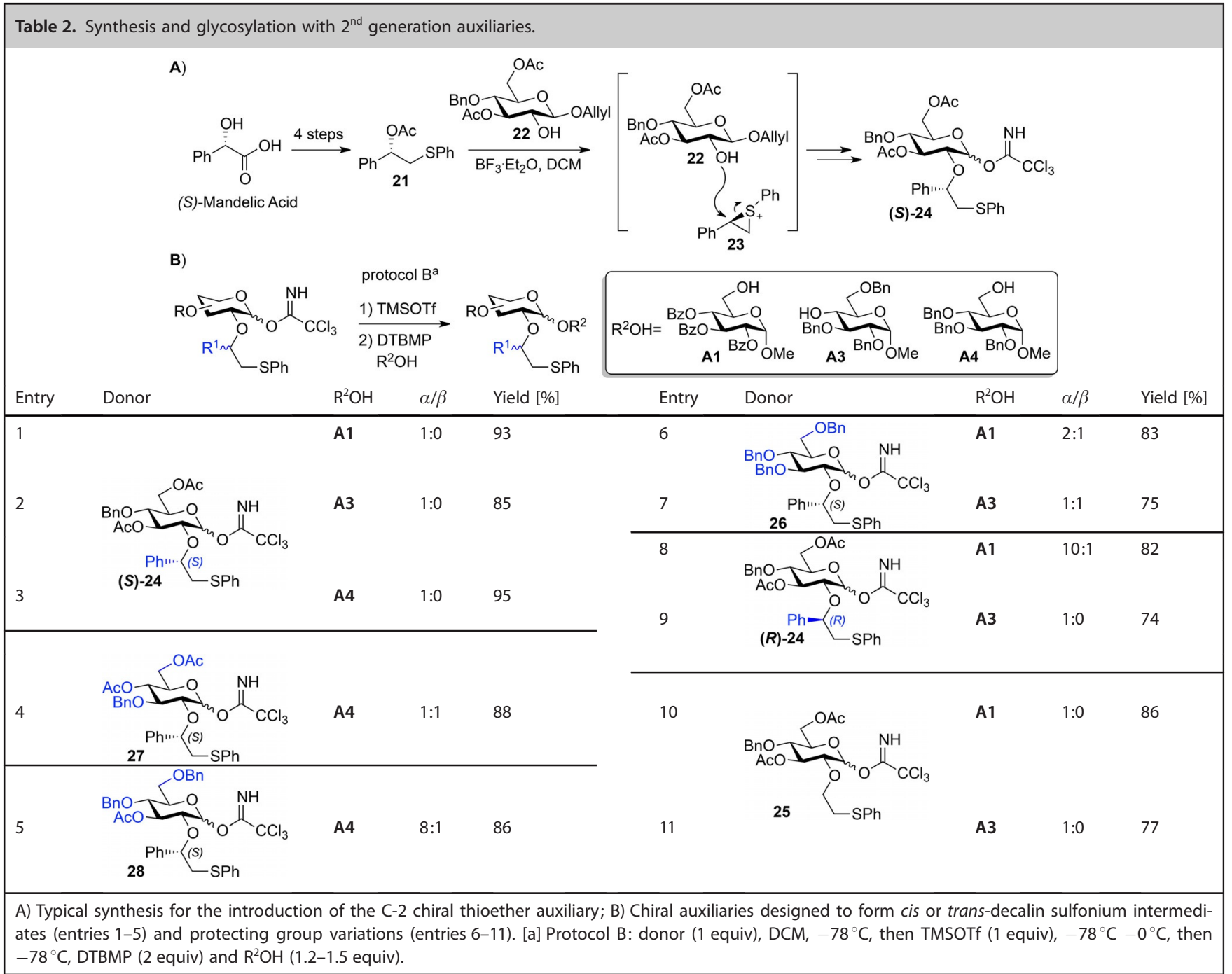

The C-2 ethoxycarbonylbenzyl moiety was installed by reaction of ethylmandelate (16) with Cerny epoxide $15^{[19]}$ and boron trifluoride (Table 1A). Subsequent acetolysis of the 1,6-anhydrobridge was carried out, followed by conversion into the trichloroacetimidate donors $(S)-18$ and $(R)-18$. Both the $R$ and $S$ isomer were prepared following this route and the resulting glycosyl imidates were evaluated as glycosyl donors (Table 1B). As expected, the use of the $S$-configured auxiliary led to the selective formation of 1,2-cis glycosides with an $\alpha / \beta$ selectivity ranging from 20:1 to 12:1 depending on the nature of glycosyl acceptor (Table 1B, entries 1-3), whereas the use of the $R$ auxiliary led to the predominate formation of 1,2-trans-glycosides (Table 1 B, entries 4-6) albeit with lower selectivity $(\alpha / \beta$ between 1:5 and 1:1 depending on the glycosyl acceptor). These results demonstrated that the chirality of the auxiliary is an important determinant of the anomeric selectivity. The protecting group pattern of the glycosyl donor was investigated in a subsequent study and found to be an important determinant of the stereoselectivity. ${ }^{[20]}$ For example, replacement of the C-3 acetyl with a benzoyl protecting group maintained $\alpha$-selectivity, yet substitution by an allyl ether diminished the $\alpha$-selectivi- ty considerably, $\alpha / \beta$ 4:1 (Table $1 \mathrm{~B}$, entries 7,8 and 9, 10, resp.). The lack of absolute stereoselectivity was attributed to glycosylation via the oxocarbenium ion. Hence, it was hypothesized that increasing the nucleophilicity of the participating functionality and reducing the flexibility of rotatable bonds in the auxiliary would speed-up neighboring group participation and improve stereoselectivity.

A second generation of chiral auxiliaries was designed to address these issues, employing a thiophenyl ether as the nucleophilic group. ${ }^{[21]}$ The (S)-(phenylthiomethyl) benzyl moiety could be stereoselectively introduced by reaction of sugar alcohol 22 with (S)-(phenylthiomethyl)benzyl acetate $\mathbf{2 1}$ in the presence of $\mathrm{BF}_{3} \cdot \mathrm{OEt}_{2}$ (Table $2 \mathrm{~A}$ ). ${ }^{[22]}$ These reactions proceed via anchimeric assistance of the thiophenyl group to form episulfonium intermediate 23 . Since double inversion occurs in this process, the stereochemistry of $\mathbf{2 1}$ is retained overall. This modification significantly improved 1,2-cis stereoselectivities and provided exclusively 1,2-cis-glycosides (Table $2 \mathrm{~B}$ ). In general, glycosylations using the (S)-(phenylthiomethyl) benzyl moiety were observed to be slower than normal indicating the presence of a quasi-stable, slow-reacting intermediate. Low 
A)

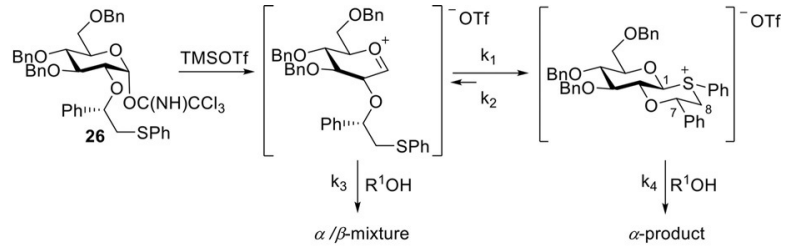

B)

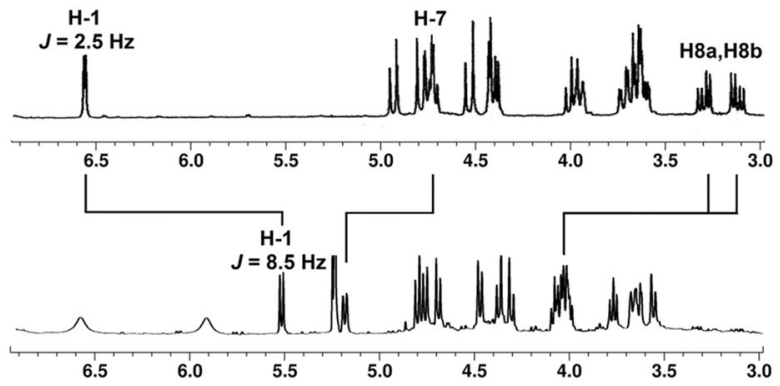

D) 8

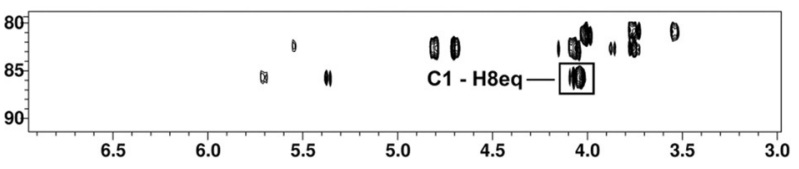

Figure $2 .{ }^{1} \mathrm{H}$ and ${ }^{13} \mathrm{C}$ NMR detection of the $\beta$-sulfonium ion intermediate. A) Curtin-Hammett principle. B) ${ }^{1} \mathrm{H}$ NMR spectrum of glucosyl donor $\mathbf{2 6}$ in $\mathrm{CD}_{2} \mathrm{Cl}_{2}$. C) ${ }^{1} \mathrm{H}$ NMR spectrum of 26 after addition of TMSOTf at $\left(-20^{\circ} \mathrm{C}\right)$. D) ${ }^{1} \mathrm{H}-{ }^{13} \mathrm{C} \mathrm{HMBC}$ spectrum of the $\beta$-sulfonium ion at $-20^{\circ} \mathrm{C}$ A cross-peak was observed between $\mathrm{C}-1$ and $\mathrm{H} 8$-eq.

temperature NMR experiments showed the selective formation of a $\beta$-linked sulfonium ion intermediate. Again, the stereoselectivity was strongly dependent on the stereochemistry of the auxiliary. As expected, the (S)-auxiliary was 1,2-cis selective whilst the $(R)$-auxiliary was non-selective (Table $2 \mathrm{~B}$, entries $1-3$ and 4 , resp.). Interestingly, an auxiliary lacking the chiral substituent was less selective than the (S)-(phenylthiomethyl) benzyl moiety, yet still gave a respectable $\alpha$-selectivity (Table 2B, entry 5). Since the 1,2-cis-decalin system experiences more unfavorable gauche interactions than the 1,2-trans-decalin system, 1,2-cis-glycosides are still formed predominantly even though no chiral substituent is present (vide infra). As in the case of the $1^{\text {st }}$ generation auxiliaries (vide supra), the stereoselectivity was also strongly dependent on the type and distribution of protecting groups on the glycosyl donor (Table 2 B, entries 6-11). ${ }^{[23]}$

For example, glycosylations of glucosyl donor (S)-24, protected with acetyl esters at C-3, and C-6, gave the corresponding glucosides as only the $\alpha$-anomer. However, similar glycosylations with glucosyl donor 26, protected with benzyl ethers gave no or very poor anomeric selectivity (Table $2 \mathrm{~B}$, entries 1 3 vs. 6,7 ). To investigate this result, extensive NMR analysis on benzyl protected donor 26 (Figure 2B) was performed to establish the presence or absence of the important $\beta$-sulfonium ion. After activation of the imidate leaving group, low-temperature NMR clearly showed the $\beta$-sulfonium ion had formed on the basis of the $\mathrm{H}-1$ chemical shift and coupling constant (Figure $2 \mathrm{C}$ ) and the correlation of $\mathrm{C}-1$ and $\mathrm{H}-8$ in the $\mathrm{HMBC}$ spectrum (Figure 2D). ${ }^{[23]}$ No other species were detected and hence the $\beta$-substituted sulfonium ion is the main reaction intermediate. It is, however, not the only reactive intermediate as addi- tion of an alcohol resulted in the formation of a mixture of anomers. This observation was rationalized by the classical Curtin-Hammett principle in which the glycosylation takes place via the much more reactive oxocarbenium ion when the rates of interconversion $\left(k_{1}\right.$ and $\left.k_{2}\right)$ are faster than that of glycosylation $\left(k_{4}\right)$ (Figure $2 \mathrm{~A}$ ). More strongly electron-withdrawing groups such as the acetyl esters are expected to disfavor oxocarbenium ion formation and hence glycosylations takes place by an $\mathrm{S}_{\mathrm{N}} 2$ like displacement of the $\beta$-anomeric sulfonium ion leading to the formation of $\alpha$-glycosides. On the other hand, glycosylations with donors having electron donating benzyl protecting groups, involve an equilibrium between the corresponding sulfonium and oxocarbenium ions. ${ }^{[2]}$ Glycosylations then take place mainly through the latter intermediate thereby diminishing the $\alpha$-selectivity. In order to establish how many, what type and at which position electron withdrawing protecting groups are needed to ensure $\alpha$-selectivity, a number of differentially protected glycosyl donors (26-28, Table 2B, entries 6-11) were synthesized and tested. From the glycosylations it is clear that one acetyl ester is sufficient to ensure stereoselective glycosylation when present at the C-3 position. ${ }^{[23]}$

To investigate this effect, DFT calculations were performed that simulated the $S_{N} 1$ and $S_{N} 2$ pathways and the role the $C-3$ acetyl plays in both (Scheme 2). ${ }^{[25]}$ These calculations showed

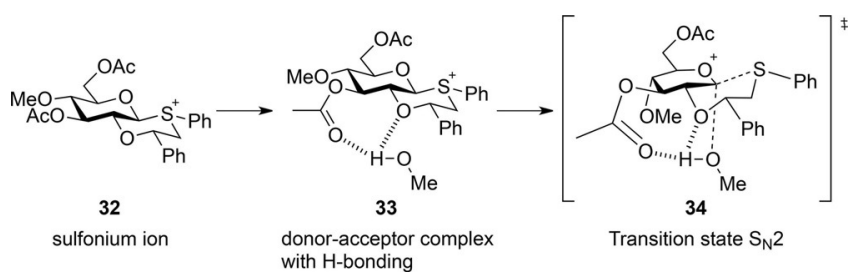

Scheme 2. Reaction intermediates according to DFT calculations of $\mathrm{S}_{\mathrm{N}} 2$ transition state involving donor-acceptor hydrogen bonding.

that the C-3 acyl group destabilizes the oxocarbenium ion in the $\mathrm{S}_{\mathrm{N}} 1$ pathway thereby promoting the $\mathrm{S}_{\mathrm{N}} 2$ pathway, as was postulated in earlier reports. ${ }^{[20,23]}$ However, the calculated energy difference between the $S_{N} 1$ and $S_{N} 2$ pathway is too small to exclude one or the other. Furthermore, DFT studies revealed that in both the $S_{N} 1$ and $S_{N} 2$ pathway, the acceptor can engage in dual hydrogen bonding with the $\mathrm{C}-3$ ester functionality and the C-2 oxygen (33) thereby directing the approach from the $\alpha$-face (34).

To further investigate if the observed intermediate sulfonium ion is a reactive intermediate or merely a resting state, the nature of the nucleophilic group was varied (Table 3$){ }^{[25]}$ Three auxiliary variants were prepared, containing either a thiophenyl,ether (29), phenyl ether (30) or benzyl (31) moiety. The $R$ and $S$-configured auxiliaries were prepared giving rise to six variants in total. From the glycosylation results, it is clear that the thiophenyl ether auxiliary performs best and the phenyl ether auxiliary only performs slightly worse. The benzyl auxiliary cannot stabilize the oxocarbenium ion and hence glycosylation through this intermediate is expected in a less selective manner. In some cases however, benzyl auxiliary containing 


\begin{tabular}{|c|c|c|c|c|c|c|c|c|c|c|c|}
\hline \multirow[b]{2}{*}{ Entry } & \multirow{2}{*}{ Donor } & \multicolumn{2}{|c|}{$\begin{array}{l}\text { protocol A } \\
\text { protocol B } \\
\text { 1) TMSOTf } \\
\text { 2) } \mathrm{R}^{1} \mathrm{OH}, \mathrm{DTBMP}\end{array}$} & \multirow{2}{*}{\multicolumn{2}{|c|}{$\alpha / \beta \quad$ Yield [\%] }} & \multicolumn{5}{|c|}{ 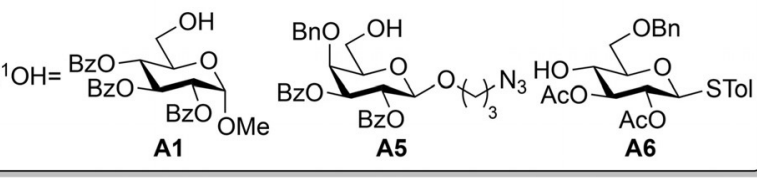 } & \multirow[b]{2}{*}{ Yield [\%] } \\
\hline & & Protocol $^{[\mathrm{a}]}$ & $\mathrm{R}^{1} \mathrm{OH}$ & & & Entry & Donor & Protocol $^{[\mathrm{a}]}$ & $\mathrm{R}^{1} \mathrm{OH}$ & $\alpha / \beta$ & \\
\hline 1 & & B & A1 & $1: 0$ & 93 & 10 & & B & A1 & $3: 1$ & 88 \\
\hline 2 & & B & A5 & $1: 0$ & 63 & 11 & & B & A5 & $3: 1$ & 70 \\
\hline 3 & $L_{-S P h}^{(1)}$ & B & A6 & 1:0 & 56 & 12 & $(R)-29$ & B & & $6: 1$ & 48 \\
\hline 4 & & A & A1 & $1: 0$ & 100 & 13 & & A & A1 & $4: 1$ & 81 \\
\hline 5 & & A & A5 & $12: 1$ & 87 & 14 & & A & A5 & $2: 1$ & 92 \\
\hline 6 & $\stackrel{L}{(S)}_{\mathrm{OPh}}$ & A & A6 & $15: 1$ & 33 & 15 & $(R)-30$ & A & A6 & 7:1 & 53 \\
\hline 7 & & A & A1 & $1: 0$ & 100 & 16 & & A & A1 & $10: 1$ & 83 \\
\hline 8 & & A & A5 & $2: 1$ & 100 & 17 & & A & A5 & $1.5: 1$ & 62 \\
\hline 9 & 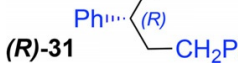 & A & A6 & $15: 1$ & 44 & 18 & (S)-31 & A & A6 & $20: 1$ & 48 \\
\hline
\end{tabular}

donor 31 performed quite well, but the same is true for a donor protected with a C-2 benzyl (data not shown, $\alpha / \beta=15: 1$ with acceptor A6). ${ }^{[2]}$ Changing the $S$-configuration to the $R$ configuration had the largest effect on the auxiliaries that are expected to perform neighboring group participation (thiophenyl ether (29) and phenyl ether (30) auxiliaries) suggesting a role of the sulfonium/oxonium ion in the reaction pathway. In case of the $R$-auxiliary, the existence of the expected $\alpha$-sulfonium ion was confirmed by NMR even though the main products of these donors were $\alpha$-glycosides. This result emphasizes that the observed intermediate is not necessarily a reactive intermediate. In case of $S$-auxiliary (S)-29, it is much more challenging to establish whether the intermediate sulfonium ion is a reactive intermediate since the observed $\alpha$-product could either be formed by $\mathrm{S}_{\mathrm{N}} 2$ displacement or highly selective alternate pathways or a combination of both. The hallmark of an $\mathrm{S}_{\mathrm{N}} 2$ reaction is the overall second order reaction kinetics, first order in both reagents. Hence, reaction rates were measured at different concentrations of glycosyl acceptor to establish the reaction kinetics. The observed initial rates of reaction could be fitted to a second order rate equation and hence these results therefore support an $\mathrm{S}_{\mathrm{N}} 2$ displacement mechanism for glycosylation. Together with the results from the DFT calculations it was concluded that glycosylation occurs via a bimolecular mechanism in an $\mathrm{S}_{\mathrm{N}} 2$-like fashion.

In addition to participation of chiral auxiliaries from the C-2 position, the participation from the $\mathrm{C}-6$ position has also been studied to prepare $\alpha$-2-deoxy sugars. ${ }^{[26]}$ Indeed the use of the C-6 auxiliary led to the stereoselective formation of $\alpha$-2-deoxyglycosides albeit with lower selectivity compared to the C-2 participation examples mentioned above.

\section{Oxathiane glycosyl donors}

Another route towards trans-decalin sulfonium ions via an integrated oxathiane stereodirecting group was developed by Turnbull et al. to reduce the synthetic effort needed for the implementation of an $\alpha$-directing moiety into the glycosyl donor (Scheme 3). ${ }^{[27]}$ It was envisioned that the thioglycoside moiety could be activated by alkylation or arylation to provide a sulfonium ion intermediate for subsequent glycosylation (Scheme 4 and 5). The oxathiane precursor $\mathbf{3 6}$ was synthesized from glucose pentaacetate by conversion into the $\beta$-thiol, addition to 2-bromoacetophenone 35 and finally deacetylation (Scheme 3). ${ }^{[27]}$ Reaction of $\mathbf{3 6}$ in acidic $\mathrm{MeOH}$ resulted in regioand stereoselective cyclization to provide methyl ketal 37, in which the smaller methoxy group is in the axial position, where it also benefits from stabilization of the anomeric effect. Hence this method obviates the need for elaborate protecting group strategies to isolate the C-2 alcohol for incorporation of the chiral auxiliary. Additionally, since the formation of the methyl ketal is stereoselective, achiral starting materials can be used to construct a chiral auxiliary.

Furthermore, the methyl ketal could be reduced in a stereoselective manner to provide oxathiane 40. Another advantage of the oxathiane donors is their stability during protecting group manipulations and the remaining triol could be protected with various protecting groups. ${ }^{[27,28]}$

The activation of oxathiane donor $\mathbf{3 9}$ was investigated by methylation using trimethyloxonium tetrafluoroborate affording 43, which proved to be highly stable and could even be crystalized from hot ethanol. ${ }^{[29]}$ The crystal structure revealed that the axial methoxy group stabilized the cation, thus 


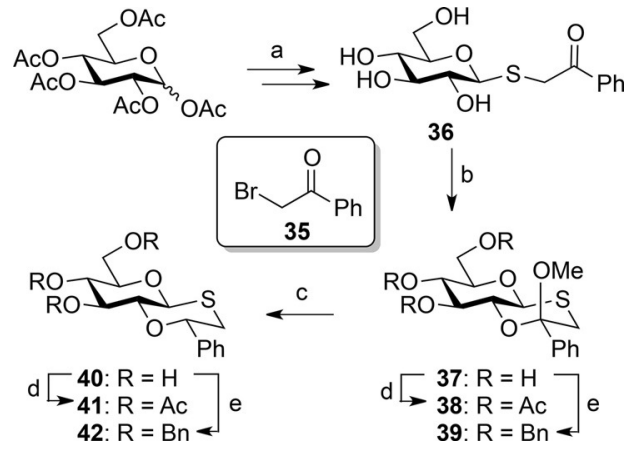

Scheme 3. Synthesis of oxathianes. Reagents and conditions: a) i) thiourea, $\mathrm{BF}_{3} \cdot \mathrm{OEt}_{2}, \mathrm{MeCN}$; ii) 35, $\mathrm{Et}_{3} \mathrm{~N}$; iii) $\mathrm{NaOMe}, \mathrm{MeOH}$; b) $\mathrm{TsOH}, \mathrm{MeOH}$; c) TMSOTf, $\mathrm{Et}_{3} \mathrm{SiH}, \mathrm{DCE} ;$ d) $\mathrm{Ac}_{2} \mathrm{O}$, pyr; e) $\mathrm{NaH}, \mathrm{BnBr}, \mathrm{DMF}$.

making it less reactive. Glycosylation of $\mathbf{4 3}$ with $\mathrm{A} 2$ was achieved in a microwave reactor at $150^{\circ} \mathrm{C}$ and after acidic cleavage of the auxiliary afforded the $\alpha$-disaccharide $\mathbf{4 4}$ with an $\alpha / \beta$ ratio of $>50: 1$ (83\%) (Scheme 4). Sulfonium ion 42, which lacks the axial methoxy group, should be less stable and hence should glycosylate with more ease. Indeed, activation of $\mathbf{4 2}$ with methyl triflate, followed by glycosylation with $\mathbf{A} 2$ at room temperature proceeded smoothly, albeit with a significant decrease in stereoselectivity $(\alpha / \beta 3: 1)$ and yield. The loss in yield was caused by competing $S$-methyl transfer from the donor salt to the newly formed $S$-methyl sulfide in the O-2 auxiliary. The loss of stereoselectivity highlights the importance of the methyl ketal.

The alkyl sulfonium ions proved to be stereoselective, yet quite unreactive and hence aryl sulfonium ions were investigated to improve the reactivity. ${ }^{[27-29]}$ Arylation of oxathiane donors can be achieved by reaction with benzyne, ${ }^{[30]}$ but the most efficient method made use of an electrophilic aromatic substitution of the sulfoxide derivative. ${ }^{[27-29]}$ meta-Chloroperoxybenzoic acid ( $m$-CPBA) was used to oxidize oxathiane 46 to afford the corresponding sulfoxide glycosyl donor 47 (Scheme 5).

Activation with $\mathrm{Tf}_{2} \mathrm{O}$ in the presence of 1,3,5-trimethoxybenzene (TMB) gave rise to arylated sulfonium ion 49 via electrophilic aromatic substitution of triflate $48 .^{[31]}$ This process re-

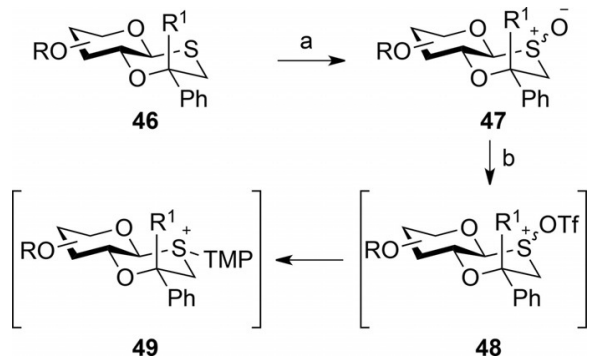

Scheme 5. Oxathiane glycosylation mechanism. Reagents and conditions: a) $\mathrm{m}$-CPBA, DCM, $-78^{\circ} \mathrm{C}$; b) $\mathrm{Tf}_{2} \mathrm{O}, 1,3,5$-trimethoxybenzene. $\mathrm{TMP}=2,4,6$-trimethoxyphenyl.

quired the use of highly electron rich aromatic compounds such as TMB and 1,3-dimethoxybenzene (DMB). The thereby formed aryl sulfonium ions proved to be much more reactive than their alkyl counterparts and also very stereoselective. In contrast to earlier auxiliaries, the methyl ketal oxathiane donors showed almost complete $\alpha$-selectivity irrespective of the protecting group pattern on the glycosyl donor (Table 4, entries 1-4). Furthermore, the stereoselectivity was excellent when galactose was used as a glycosyl donor (Table 4, entries 5,6$).{ }^{[27]}$

The only drawback of the methyl ketal donors is that the acyclic methyl ketal formed after glycosylation proved to be labile, leading to the release of methanol which subsequently acted as a competing nucleophile. Additionally, the workup procedure resulted in further loss of the ketal functionality, thus the auxiliary was removed completely with $\mathrm{BF}_{3} \cdot \mathrm{OEt}_{2}$ in methanol prior to workup. To address this issue the more stable ether oxathiane donors $\mathbf{5 4}$ and $\mathbf{5 5}$ were investigated. ${ }^{[28-30]}$ These donors proved to be more stable and provided disaccharides in higher yields. However, the stereoselectivity was reduced compared to the methyl ketal donors again highlighting the importance of this functionality. Whilst the methyl ketal donors provided good stereoselectivity in either acetylated (50) or benzylated (51) form, the ether variants (54 and 55) proved much more sensitive to the protecting group pattern on the donor. ${ }^{[28,29]}$ This was further investigated by the synthesis and glycosylation of differentially protected oxathiane donors 56-61 (Table 4, entries 11-20). In general, the use of electron withdrawing groups, especially at C-3, proved to be important to maintain $\alpha$-selectivity which is in good agreement with aforementioned results. Additionally, temperature might also play an important role in the glycosylations as protocol D adds the acceptors at $-10^{\circ} \mathrm{C}$, whereas protocol $\mathrm{E}$ adds the acceptors at $-40^{\circ} \mathrm{C}$ and the stereoselectivity is much higher in the latter protocol The mechanism of glycosylation of oxathiane donors was also investigated in silico using DFT calculations to establish the role of the sulfonium ion in the reaction pathway. ${ }^{[29]}$ The $S_{N} 1$ and $S_{N} 2$ pathways of both ketal and ether sulfonium functionalities were investigated, but neither was in full agreement with the experimental results. It is however clear that the sulfonium
Scheme 4. $\alpha$-selective glycosylations of surprisingly stable activated oxathiane $\mathbf{4 3}$ and in situ activation of donor 42. alow yield was caused by competing S-methyl transfer from the donor salt to the newly formed S-methyl sulfide in the $0-2$ auxiliary $C S A=(1 R)-(-)$ camphorsulfonic acid.
1) MeOTf, rt, $4 \mathrm{~h}$

2) A2, DTMBP, rt, $18 \mathrm{~h}$ 


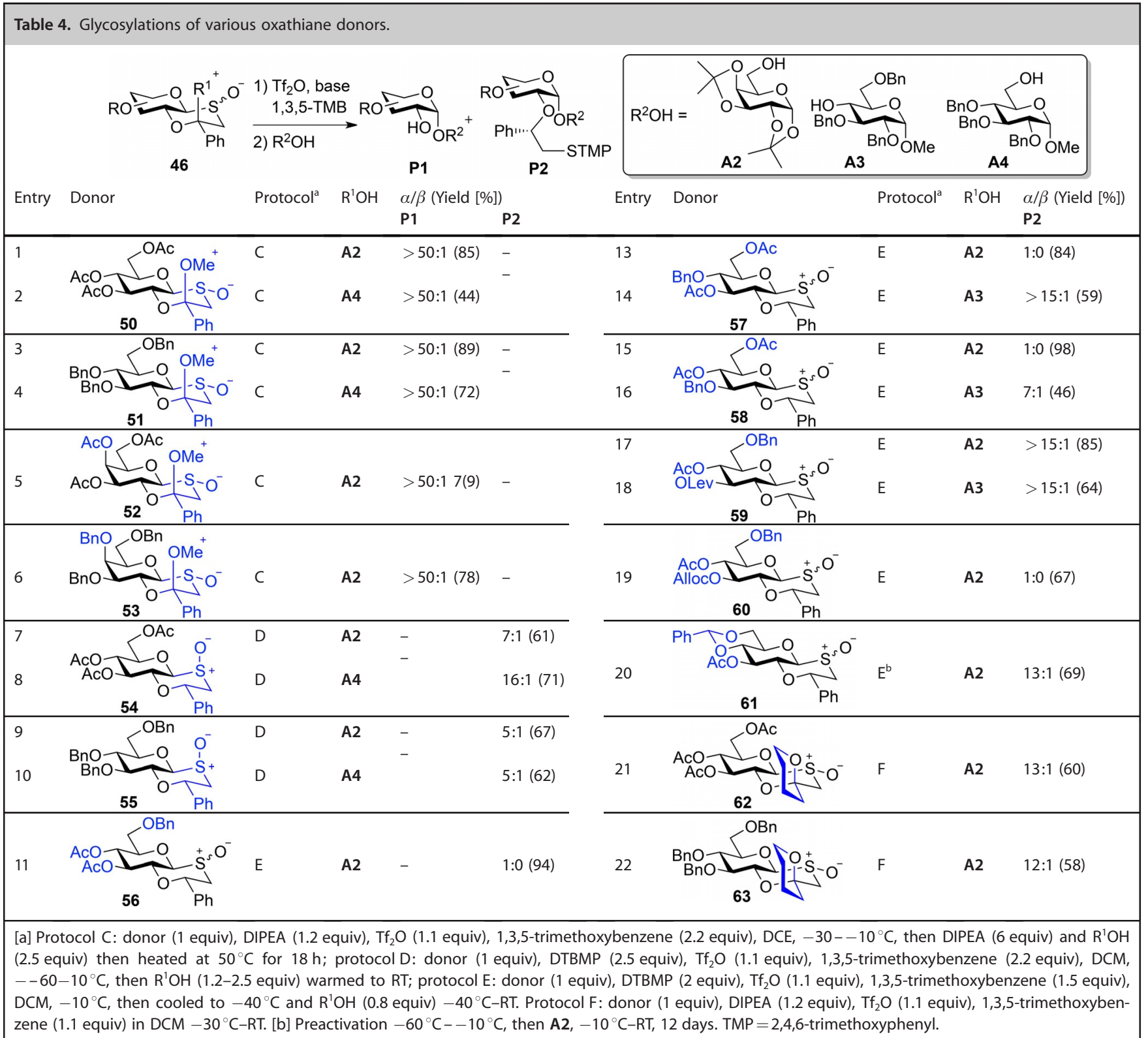

ion does probably not react solely via an $\mathrm{S}_{\mathrm{N}} 2$ mechanism on which basis these glycosyl donors were designed.

From the experimental results it was clear that the methyl ketal was vital for excellent stereoselectivity, but proved unstable after glycosylation. With this in mind, Turnbull et al. set out to improve the ketal stability by introducing a spiroketal $(62$ and 63$).{ }^{[32]}$ This auxiliary conserves the cyclic nature even after it is displaced by the glycosyl acceptor, hence is more stable and cannot release a competing nucleophile. The spiroketal oxathianes 62 and 63 were prepared starting from a dihydropyran precursor using a similar strategy as used for the methyl ketals. Activation was achieved by oxidation to the sulfoxide and reaction with TMB followed by glycosylation. The reactions proceeded well with a lower acceptor concentration as intended since no competing nucleophile was released. The isolation of the disaccharides again proved to be more convenient after acidic removal of the ketal auxiliary. The glycosylation results reveal a loss of stereoselectivity with the spiroketal, $\alpha / \beta$ 13:1 (Table 4, entry 21, acetylated donor) and $\alpha / \beta$ 12:1 (Table 4, entry 22, benzylated donor) compared to their methyl ketal counterparts $(\alpha / \beta>50: 1$, Table 4, entries 1 and 3) suggesting that the structure of the ketal is important for the stereoselectivity. It was noted that the glycosylation reaction was sluggish and tuning of the reactivity of the sulfonium ion by modifying the aryl structure was investigated. It was envisioned that the use of less electron-rich DMB would lead to a less stabilized and therefore more reactive sulfonium ion. A competition experiment between oxathiane 62 activated with $\mathrm{TMB}$ or $\mathrm{DMB}$ showed that the $\mathrm{DMB}$ was indeed $\approx 1.5$ times more reactive. However, the increase in reactivity led to a decrease in selectivity in case of spiroketal donor $\mathbf{6 2}$ which is consistent with earlier findings. 


\section{Achiral Auxiliaries for Glucosides and Galacto- sides}

The observation made by Boons et al. that the achiral version of their auxiliary still provided mainly 1,2-cis-products $(\alpha / \beta$ 8:1, Table 2B, entry 5) inspired researchers to investigate the use of achiral auxiliaries as they are simpler to synthesize and do not require chiral starting materials. Fairbanks et al. investigated the role of the nucleophilic group in such achiral auxiliaries and variants containing a thiophene, ${ }^{[33]}$ iodo and selenophen$\mathrm{yl}^{[34]}$ or 2,4,6-trimethoxythiophenyl group (Scheme 6). ${ }^{[35]}$ Alkyla-

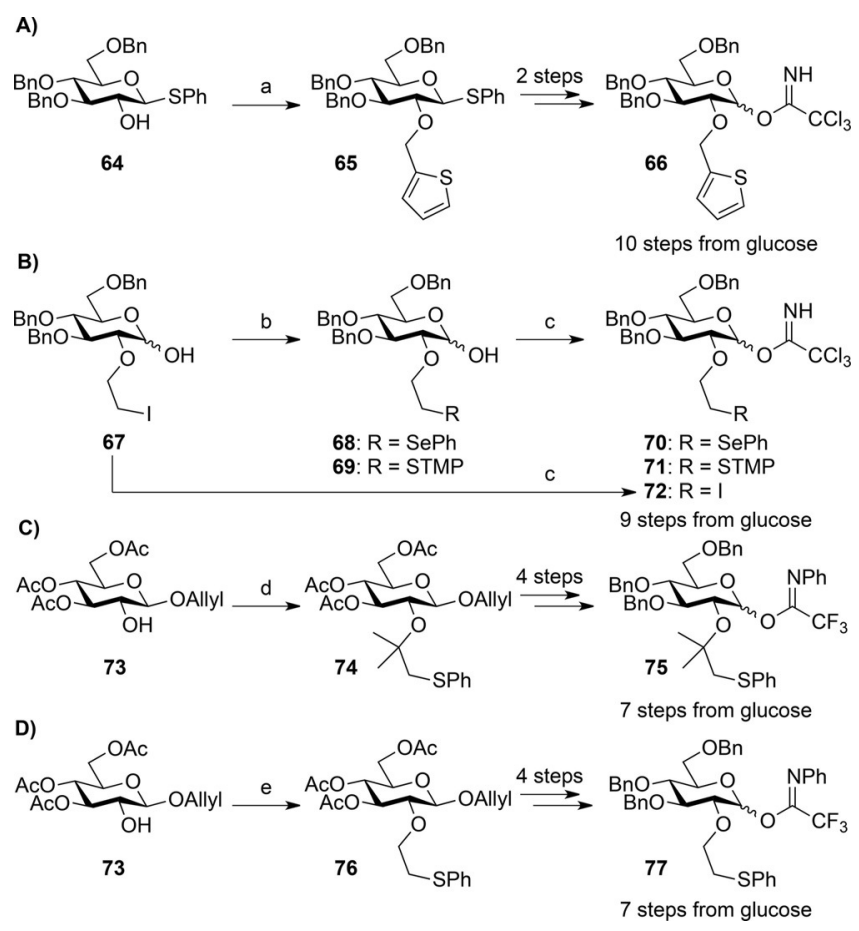

Scheme 6. Building block syntheses containing the achiral auxiliary A) thiophene, B) selenium, iodide and sulfur C) sulfur, including the Thorpe-Ingold effect D) sulfur. Conditions and reagents a) 2-(bromomethyl)thiophene, $\mathrm{NaH}$, DMF; b) PhSeH or 2,4,6-trimethoxybenzenethiol, DIPEA, DMF; c) $\mathrm{Cl}_{3} \mathrm{CCN}$, $\mathrm{DBU}, \mathrm{DCM}, 0^{\circ} \mathrm{C}$; d) $\mathrm{HO}\left(\mathrm{CH}_{3}\right)_{2} \mathrm{CCH}_{2} \mathrm{SPh}, \mathrm{BF}_{3} \cdot \mathrm{OEt}_{2}, \mathrm{DCM}, 0^{\circ} \mathrm{C}$; e) $\mathrm{Br}\left(\mathrm{CH}_{2}\right)_{2} \mathrm{SPh}$, $\mathrm{Ag}_{2} \mathrm{CO}_{3}, \mathrm{AgClO}_{4}$, toluene, $60^{\circ} \mathrm{C}$. DIPEA $=N, N$-Diisopropylethylamine, $\mathrm{DBU}=1,8$-diazabicyclo[5.4.0]undec-7-ene.

tion of the C-2 position in 64 with 2-bromomethyl thiophene under standard conditions gave intermediate 65 which could be converted into glycosyl imidate 66 (Scheme $6 \mathrm{~A}) .^{[33]}$ The installation of the 2-(iodo)ethyl-, 2-(selenophenyl)ethyl- and 2(2,4,6-trimethoxythiophenyl)ethyl ethers proved more challenging than expected. The best route relied on the 2-iodo-ethyl precursor 67 which was prepared by the conversion of a C-2 allyl ether via ozonolysis, reduction and subsequent iodination of the alcohol (Scheme 6B). ${ }^{[34]}$ Conversion of this intermediate into the glycosyl imidate afforded the 2-(iodo)ethyl auxiliary donor 72. Nucleophilic displacement of the iodide using phenyl selenol or 2,4,6-trimethoxythiophenol afforded hemiacetals 68 and 69 and subsequent imidation gave glycosyl donors 70 and $\mathbf{7 1} .^{[34,35]}$ Using this chemistry, a number of differentially protected glycosyl donors were prepared.
Inspired by the success of the methyl ketal oxathiane donors (vide supra), the role of the geminal substituents on the auxiliary was investigated. ${ }^{[36]}$ It was shown that the methyl ketal is crucial for stereoselectivity as it is able to stabilize the sulfonium ion using electron density of the methoxy substituent. Additionally, the presence of two geminal substituents is also known to speed-up ring closure via the Thorpe-Ingold, or gem-dialkyl, effect. ${ }^{[37]}$ This could also be a major factor that improves the methyl ketal donors stereoselectivity. To investigate this further, achiral variants containing a geminal dimethyl substituent were prepared. Starting from C-2 alcohol precursor $73{ }^{[38]}$ the auxiliary was installed by reaction of the corresponding tertiary alcohol and $\mathrm{BF}_{3} \cdot \mathrm{Et}_{2} \mathrm{O}$ (Scheme $6 \mathrm{C}$ ). To establish the effect of the gem-dimethyl substituent, a set of control compounds lacking this substituent were also prepared. To this end, the same precursor (73) could be alkylated using 2-(bromoethyl)thiophenyl ether and silver perchlorate (Scheme 6D). The same chemistry was applied to prepare galactoside donors as well. ${ }^{[36]}$

The glycosylation results of glycosyl donors carrying achiral auxiliaries are summarized in Table 5 . The stereoselectivity with the thiophene based auxiliary was poor to moderate depending on the type of protecting groups used. ${ }^{[33]}$ Acetylated donor 78 was unselective $(\alpha / \beta$ 1.4:1) and the benzylated counterpart (66) was much more stereoselective $(\alpha / \beta$ 9:1). In the latter case, the stereoselectivity was also strongly dependent on the type of glycosyl acceptor (up to $\alpha / \beta 30: 1$ for benzylidene-protected mannose acceptors). ${ }^{[33 a]}$ In the case of both the acetyland benzyl-protected donor, low-temperature NMR studies showed the presence of distorted $\beta$-configured sulfonium intermediates. ${ }^{[33 b]}$ From the glycosylation results it is clear however, that these intermediates are probably not reactive intermediates.

In a subsequent study, the use of a 2-(iodo)ethyl or 2-(selenophenyl)ethyl ether as a participating group was explored. ${ }^{[34]}$ Very poor selectivity was observed in both cases with again the benzylated glycosyl donors performing better than their acetylated counterparts (Table 5 , entries 2,3 vs. 10,11). This result is the reverse of what would be expected based on the findings in earlier studies that show that electron-withdrawing groups on the glycosyl donor perform better than electron-donating ones. ${ }^{[23]}$ Again, low-temperature NMR experiments were performed to detect reaction intermediates. In the case of the 2-(iodo)ethyl auxiliary no iodonium ion could be detected for acetylated donor $\mathbf{7 9}$ or benzylated donor $\mathbf{7 2}$. Hence, it is clear that this group does not participate in the reaction. In the case of the 2-(selenophenyl)ethyl auxiliary, a selenium ion was only detected in the case of acetylated donor $\mathbf{8 0}$. However, the stereoselectivity of donor $\mathbf{8 0}$ is lower than the benzylated counterpart (70) which does not show selenium ion formation in the variable temperature (VT) NMR studies. Hence in this case it is also unlikely that the observed stereoselectivity is a result of neighboring group participation.

As a result, more electron-rich participating groups were explored next. Inspired by the work of Turnbull et al. the 2,4,6-trimethoxythiophenyl participating group was explored. ${ }^{[35]}$ This auxiliary showed much improved stereoselectivities with the 


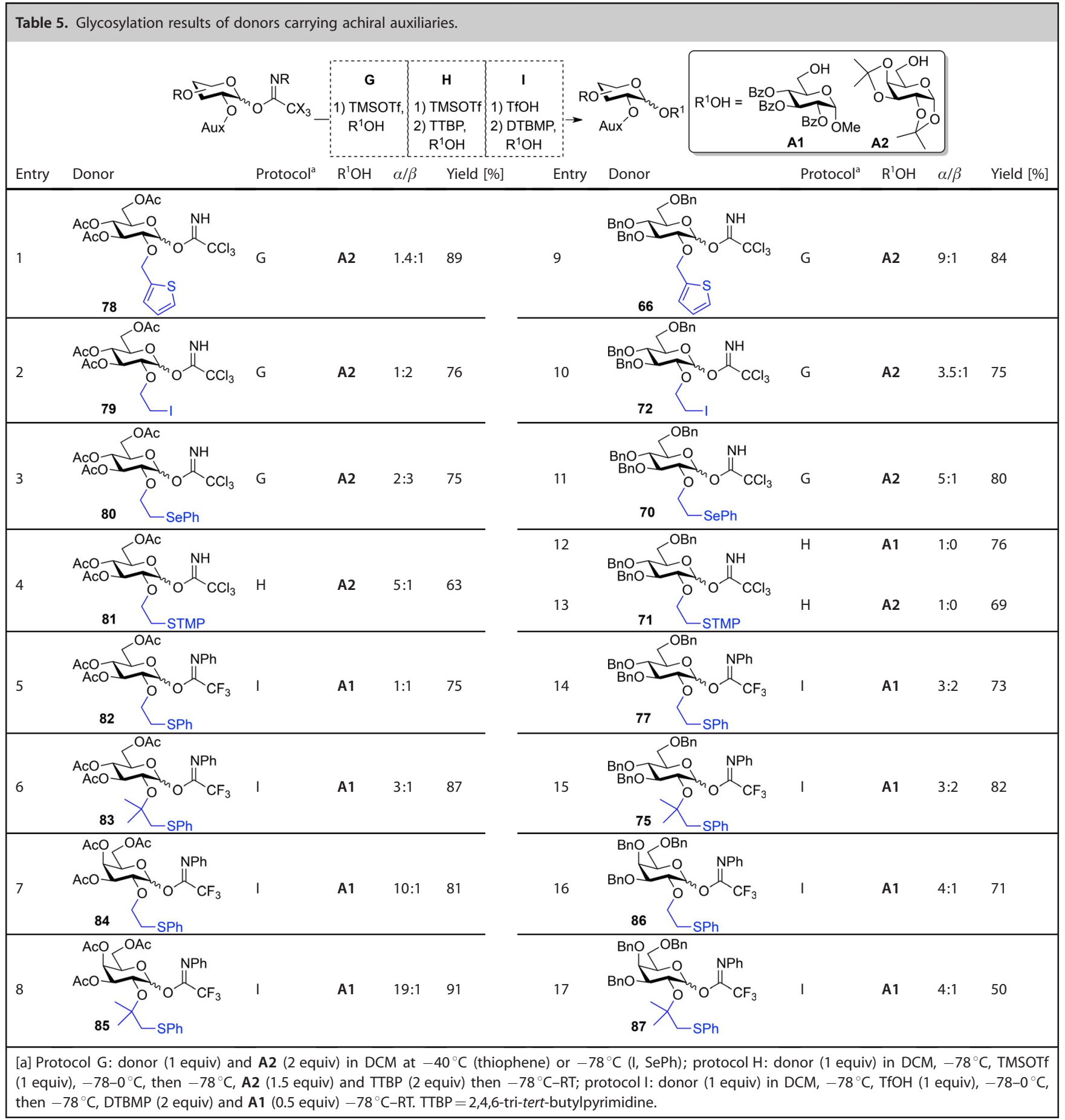

acetylated donor (81) being moderately selective $(\alpha / \beta, 5: 1)$ and benzylated donor $\mathbf{7 1}$ being fully $\alpha$-selective for a range of glycosyl acceptors. This surprising result was rationalized by NMR experiments which showed the formation of a $\beta$-sulfonium ion in case of benzylated donor 71, but not in the case of acetylated counterpart $\mathbf{8 1}$. This result is surprising considering that similar chiral systems were much less selective. ${ }^{[23]}$

Another set of achiral auxiliaries was investigated that were designed to improve the stereoselectivity on the basis of the Thorpe-Ingold effect. ${ }^{[36,37]}$ To this end, auxiliaries carrying a gem-dimethyl group were evaluated on both glucose and gal- actose in acetylated ( 83 and 85 , respectively) and benzylated form ( $\mathbf{7 5}$ and $\mathbf{8 7}$, respectively). In addition, to probe the effect of the gem-dimethyl group, a set of control compounds lacking this functionality were also investigated. In all cases the benzylated donors performed worse than the acetylated versions, which is in line with earlier findings (vide supra) (Table 5 , entries 5-8 vs. 14-17). The stereoselectivity was low to moderate with the exception of the acetylated galactose donors. In these cases, good (10:1) to excellent (19:1) stereoselectivities were found for both the unsubstituted (84) and gem-dimethylsubstituted auxiliary (85), respectively. VT-NMR studies on the 
reaction intermediates showed the formation of sulfonium ions in all cases. Glycosyl donors containing the unsubstituted auxiliary showed a mixture of $\alpha$ - and $\beta$-sulfonium ions, which upon warming, equilibrated towards the $\beta$-sulfonium ion. The same holds for the gem-dimethyl auxiliary, and it was observed that the equilibration was faster for galactose as compared to the glucose donors. Although galactosyl donor $\mathbf{8 5}$ was very stereoselective, it is difficult to determine the reason for the highly $\alpha$-selective glycosylation. VT-NMR experiments indicate temperature dependent conversion of the $\alpha$-sulfonium ion to the $\beta$-sulfonium ion followed by stereoselective glycosylation indicating that $\mathrm{S}_{\mathrm{N}}$ 2-like displacement may be responsible for the observed $\alpha$-selectivity. However, the $\alpha$-selectivity could also be the result of a more selective alternative pathway for example, remote participation. ${ }^{[39]}$

The use of achiral auxiliaries has potential as evidenced by the use of the 2-(2,4,6-trimethoxythiophenyl)ethyl auxiliary (71) which was highly stereoselective. However, the synthesis of glycosyl donors containing such auxiliaries is still quite lengthy and does not offset the benefit of not having to use chiral starting materials. Furthermore, the identification of the unwanted $\alpha$-sulfonium ions in some cases indicate that a chiral substituent is critical in directing the approach of the nucleophilic participating group.

\section{Chiral Auxiliaries for Mannosides}

As seen above, chiral auxiliaries have great potential to stereoselectively provide 1,2-cis-glucosides and 1,2-cis-galactosides (Scheme $7 \mathrm{~A}$ ). It is difficult to use this methodology in mannotype sugars since they contain an axially oriented C-2 substituent. The trans-decalin sulfonium ion intermediate is therefore unlikely formed in mannose and the most likely intermediate would be a cis-decalin system which would provide the 1,2trans-mannoside instead (Scheme 7B). Very recently, a solution

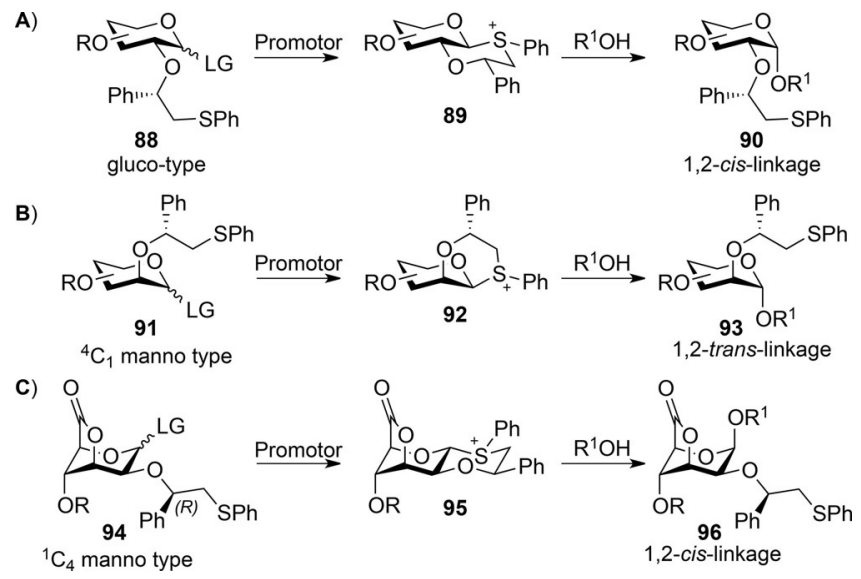

Scheme 7. General overview of NGP in manno- and glucotype donors: A) neighboring group participation by a chiral auxiliary in gluco- and galactotype donors resulting in the 1,2-cis product; B) C-2 participation of the auxiliary in the ${ }^{4} C_{1}$ conformation results in the cis-decalin system and hence 1,2-trans-mannosides; C) A ring-flip into the ${ }^{1} C_{4}$ conformation favors the trans-decalin system and glycosylation affords the $\beta$-mannoside. $L G=$ leaving group. to this issue was reported by the use of mannosides locked the ${ }^{1} \mathrm{C}_{4}$ conformation. ${ }^{[10 \mathrm{~b}]}$ In this conformation, and by using an $(R)$-configured auxiliary, the formation of a trans-decalin intermediate 95 was envisioned to be possible as the ring-flip achieves the prerequisite 1,2-trans-diequatorial stereochemistry in the sulfonium ion stage (Scheme 7C).

To this end, three mannosyl donors, 102, 104, and 105 were prepared to study the effect of conformation upon neighboring group participation and the stereoselectivity of the ensuing glycosylation (Scheme 8). ${ }^{[10 b]}$ These donors were prepared from

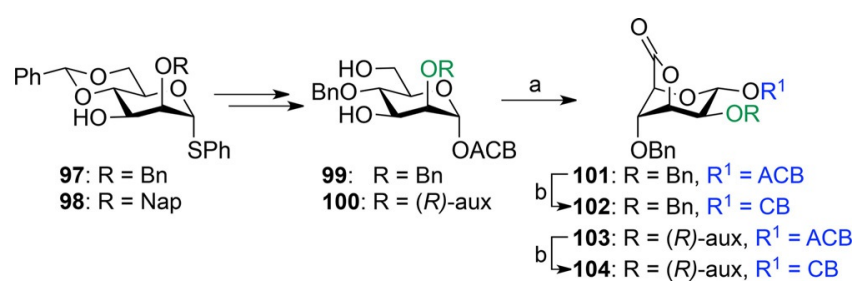

Scheme 8. Mannose lactone building block synthesis. Reagents and conditions: a) TEMPO, BAIB, $\mathrm{H}_{2} \mathrm{O} / \mathrm{DCM}(1: 10)$, b) $\mathrm{Pd}\left(\mathrm{PPh}_{3}\right)_{4}, \mathrm{AcOH} / \mathrm{DCM} . \mathrm{ACB}=$ allylcarboxybenzyl, $\mathrm{BIAB}=$ bis-acetoxyiodobenzene, $\mathrm{CB}=$ carboxybenzyl, Nap = 2methylnaphthyl, $(R)-\mathrm{Aux}=(R)-\mathrm{CH}(\mathrm{Ph}) \mathrm{CH}_{2} \mathrm{SPh}$.

two thiomannoside intermediates (97 and 98), to which an anomeric carboxybenzyl leaving group was introduced. ${ }^{[40]}$ The more common imidate leaving groups are not compatible with these lactone donors as the prerequisite lactol undergoes ring opening. The auxiliary was introduced using $(R)$-1-phenyl2-(phenylthio)ethyl acetate and $\mathrm{BF}_{3} \cdot \mathrm{OEt}_{2}{ }^{[21]}$ and to establish the contribution of the auxiliary, a control donor containing a C-2 benzyl ether was also prepared. After deacetylation to afford 3,6-diols 99 and 100, a TEMPO (2,2,6,6-tetramethylpiperidinyloxyl) oxidation was carried out to achieve the ring flip to the ${ }^{1} \mathrm{C}_{4}$ conformation. Critically, this mild oxidation method is chemoselective and compatible with thioethers. ${ }^{[41]}$ Finally, removal of the allyl ester rendered the carboxybenzyl amendable to activation and glycosylation.

Glycosylation was achieved by activation with $\mathrm{Tf}_{2} \mathrm{O}$ in the presence of 2,6-di-tert-butyl-4-methylpyridine (DTBMP) and subsequent addition of acceptor. ${ }^{[40]}$ The glycosylation results reveal that the ${ }^{4} C_{1}$ mannoside 105 produced mainly $\alpha$-mannosides, presumably via previously mentioned cis-decalin sulfonium ion intermediate 92 (Table 6, entries 1-2). In contrast, glycosylation with 104 showed excellent $\beta$-selectivity in respectable yields (Table 6 , entries 3,4 ). The $\beta$-selectivity observed in these glycosylation supports the hypothesis that the reaction proceeds via an $\mathrm{S}_{\mathrm{N}}$ 2-like displacement of the proposed equatorial sulfonium ion. However, control donor 102, also produced exclusively $\beta$-mannosides with both the primary and secondary acceptor (Table 6, entries 5-6). These results indicate that reactive intermediates other than the $\alpha$-sulfonium ions are also important for the observed $\beta$-selectivity.

To explain the results, VT-NMR studies were performed on donors 105 and 104 to identify reaction intermediates. Lowtemperature NMR experiments of donor 104 clearly showed the presence of the expected $\alpha$-sulfonium ion on the basis of 


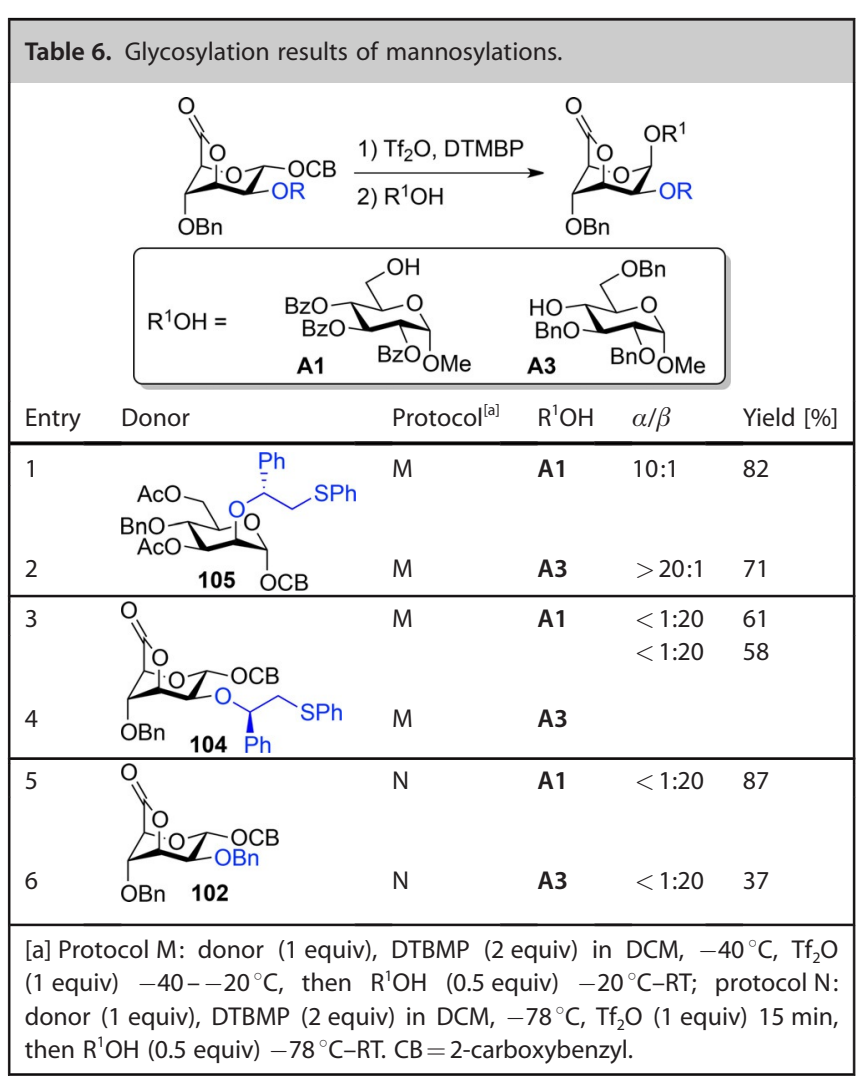

a strong correlation between $\mathrm{C}-1$ and $\mathrm{H}-8_{\mathrm{ax}}(\mathrm{HMBC})$ and the ${ }^{3} J_{\mathrm{H} 1-\mathrm{H} 2}$ coupling of $6.0 \mathrm{~Hz}$, which supports a diaxial orientation. The observation of the $\beta$-sulfonium ion after activation of 105 and the $\alpha$-sulfonium ion found following activation of 104 support the hypothesis that these intermediates are displaced by the alcohol to provide $\alpha$ - and $\beta$-mannosides, respectively. Glycosylation with mannosyl donor 102, however, were also highly $\beta$-selective. Hence, the reaction intermediates formed after activation of donor 102 were also investigated using VTNMR. Low temperature $\left(-78^{\circ} \mathrm{C}\right)$ spectra showed a mixture of species, but as the temperature was raised one species remained at $-20^{\circ} \mathrm{C}$. Extensive NMR studies showed a strong $\mathrm{H} 1-\mathrm{C} 4$ correlation which, together with the formation of benzyl triflate, led to the conclusion that tricyclic compound 107 had been formed (Scheme 9). The formation of 107 is proposed to proceed via stabilization of the intermediate oxocarbenium ion 109 by the C-4 benzyl ether to form 112 (Scheme 9). In absence of the acceptor, this intermediate ultimately results in the formation of 107 and benzyl triflate. The formation of 107 may also explain the high $\beta$-selectivity of 102. Careful examination of the glycosylation mixture indeed showed the presence of $\mathbf{1 0 7}$ as a side product in moderate quantities. A batch experiment to produce 107 from 102 afforded tricyclic compound 107 in good yield (79\%). In addition to remote participation of the C-4 benzyl ether, the expected ${ }^{3} \mathrm{H}_{4}$ conformation of the oxocarbenium ion may also lead to $\beta$ product (Scheme 9). In principle, although not observed in the NMR study at $-20^{\circ} \mathrm{C}, \mathrm{C}-4$ benzyl participation could also occur in 104 and indeed tricyclic compound 113 could be identified

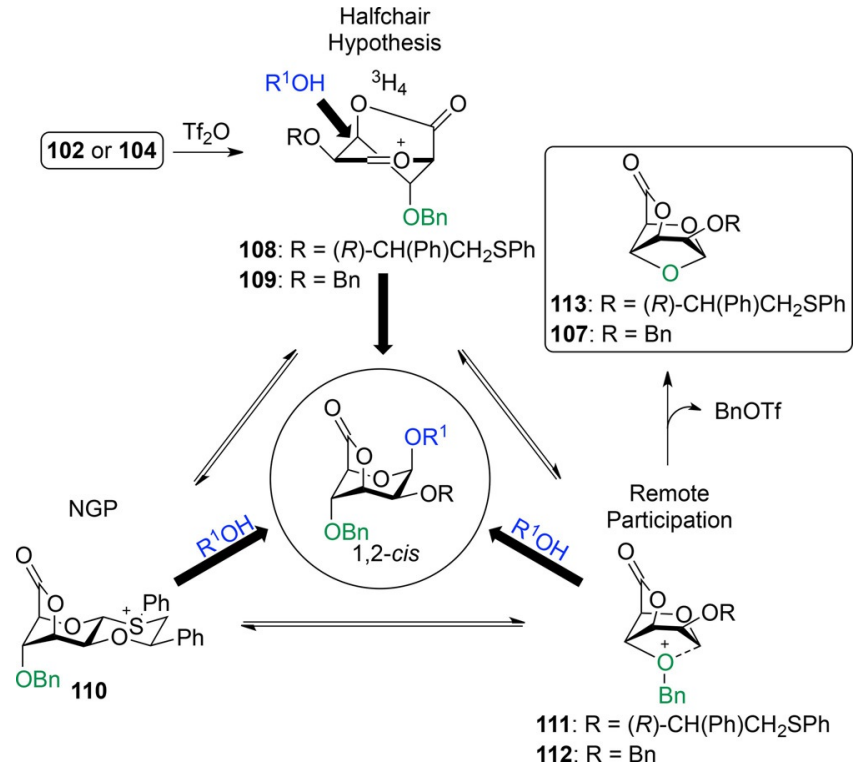

Scheme 9. Proposed pathways for remote participation in mannuronic acid lactones 102 and 104.

as a side product in the glycosylation reaction. However, although $\mathbf{1 1 3}$ is ultimately formed, VT-NMR experiments showed that sulfonium ion formation was highly favored at $-20^{\circ} \mathrm{C}$. Therefore, in the case of 104 an additional pathway via sulfonium ion $\mathbf{1 1 0}$ may lead to $\beta$-mannosides, but the observed selectivity is more likely to be a result of the aforementioned alternative pathways (Scheme 9).

\section{Application to Complex Oligosaccharide Synthesis}

The true scope and limitations of synthetic methodologies are best established by their application in total syntheses. As can be seen in the previous sections, the protecting groups on the glycosyl donor and its relative stereochemistry as well as the structure of the auxiliary have a profound influence on the stereoselectivity. Application to total synthesis has revealed other aspects to keep in mind when using chiral auxiliaries such as protecting group compatibility, auxiliary removal, sterics and its potential with respect to solid-phase synthesis. To date, three total syntheses of oligosaccharides using chiral auxiliaries have been reported. ${ }^{[28,42]}$ Absolute stereocontrol is especially important to enable solid phase oligosaccharide synthesis. Solid-phase oligosaccharide synthesis (SPOS) offers the promise of increasing the speed of oligosaccharide assembly, primarily by eliminating intermediate purification steps and by automation. ${ }^{[43]}$ Since no intermediate purification is undertaken, intractable mixtures of compounds will be formed if the glycosylations are not fully stereoselective. Not surprisingly, only a few examples of SPOS of oligosaccharides containing 1,2-cis-glycosides have been reported. ${ }^{[44]}$ These rely on tedious separation of the anomers by high-performance liquid chromatography (HPLC) or the preparation of a 1,2-cis-linked disaccharide in solution, which after purification to remove the unwanted 1,2- 
trans-anomer, can be used in solid-phase synthesis. Thus, a major stumbling block in SPOS is the inability to reliably introduce 1,2-cis-glycosides with complete stereoselectivity.

The use of chiral auxiliaries to address this issue was explored in the synthesis of a branched $\alpha$-linked pentasaccharide found in Aconitum carmichaeli that has the potential to be developed as an adjuvant. ${ }^{[45]}$ The polysaccharide is composed of an $\alpha(1,6)$-linked glucosyl backbone, branched with an $\alpha(1,3)$ linked glucoside moiety. Such $\alpha$-glucans are considerably challenging to synthesize on solid supports due to the multitude of 1,2-cis-linkages and their branched structure. Pentasaccharide 121 from Aconitum carmichaeli was prepared from monosaccharide building blocks 115, (S)-24 and 118 (Scheme 10). ${ }^{[42 a]}$ By using orthogonal allyloxycarbonyl (Alloc) and fluorenylmethyloxycarbonyl (Fmoc) protecting groups, branching could be achieved. The Fmoc protecting group of $\mathbf{1 1 4}$ was removed by treatment with piperidine/DMF and coupled with auxiliary-containing glucosyl donor $\mathbf{1 1 5}$ to install the first 1,2-cis linkage. The couplings were performed by the addition of preformed sulfonium ion to the acceptor. Double couplings were performed to achieve full conversion. The Alloc function could be removed by treatment with $\mathrm{Pd}\left(\mathrm{PPh}_{3}\right)_{4}$ in a mixture of THF and $\mathrm{AcOH}$ to give the corresponding disaccharide acceptor. However, this acceptor could not be coupled with preactivated (S)24. It was hypothesized that the $\mathrm{C}^{\prime}$ hydroxyl is sterically shielded by the neighboring (S)-(phenylthiomethyl)benzyl ether. Thus, the auxiliary was converted into an acetyl ester by treatment with acetic anhydride in the presence of $\mathrm{BF}_{3} \cdot \mathrm{OEt}_{2}$ affording 116. The Alloc was removed under standard conditions and coupling with pre-activated (S)-24 was now successful to yield resin-bound trisaccharide 117. A sequence of Fmoc removals and couplings gave rise to the complete protected pentasaccharide 119 . The C-2 auxiliaries were removed onresin under aforementioned conditions and the product was cleaved using $\mathrm{NaOMe} / \mathrm{MeOH}$ affording pentasaccharide 120. Reacetylation was performed to aid purification and NMR analysis. No anomeric isomers of the completed product were detected which was obtained in an overall yield of $25 \%(90 \%$ per step, 13 on-resin steps). Finally, target compound 121 was prepared by removal of the acetyl esters, using standard conditions followed by hydrogenolysis of the benzyl ethers using $\mathrm{Pd}(\mathrm{OH})_{2} / \mathrm{C}(20 \mathrm{wt} \%)$ and $\mathrm{H}_{2}$ gas.

The total synthesis of pentasaccharide 121 was the first example of a stereoselective solid-supported synthesis of an oligosaccharide having multiple 1,2-cis-glycosidic linkages. A particularly interesting feature was that a relatively small excess (2.0 equiv) of glycosyl donor was required to drive the glycosylations to completion. It is likely that the intermediate sulfonium ion is sufficiently stable to diffuse into the polymer support for glycosylation of the resin-bound sugar hydroxyls. However, a limitation of the C-2 auxiliary is that it may block the neighboring C-3 hydroxyl from reacting and hence requiring auxiliary removal prior to glycosylation at this site.

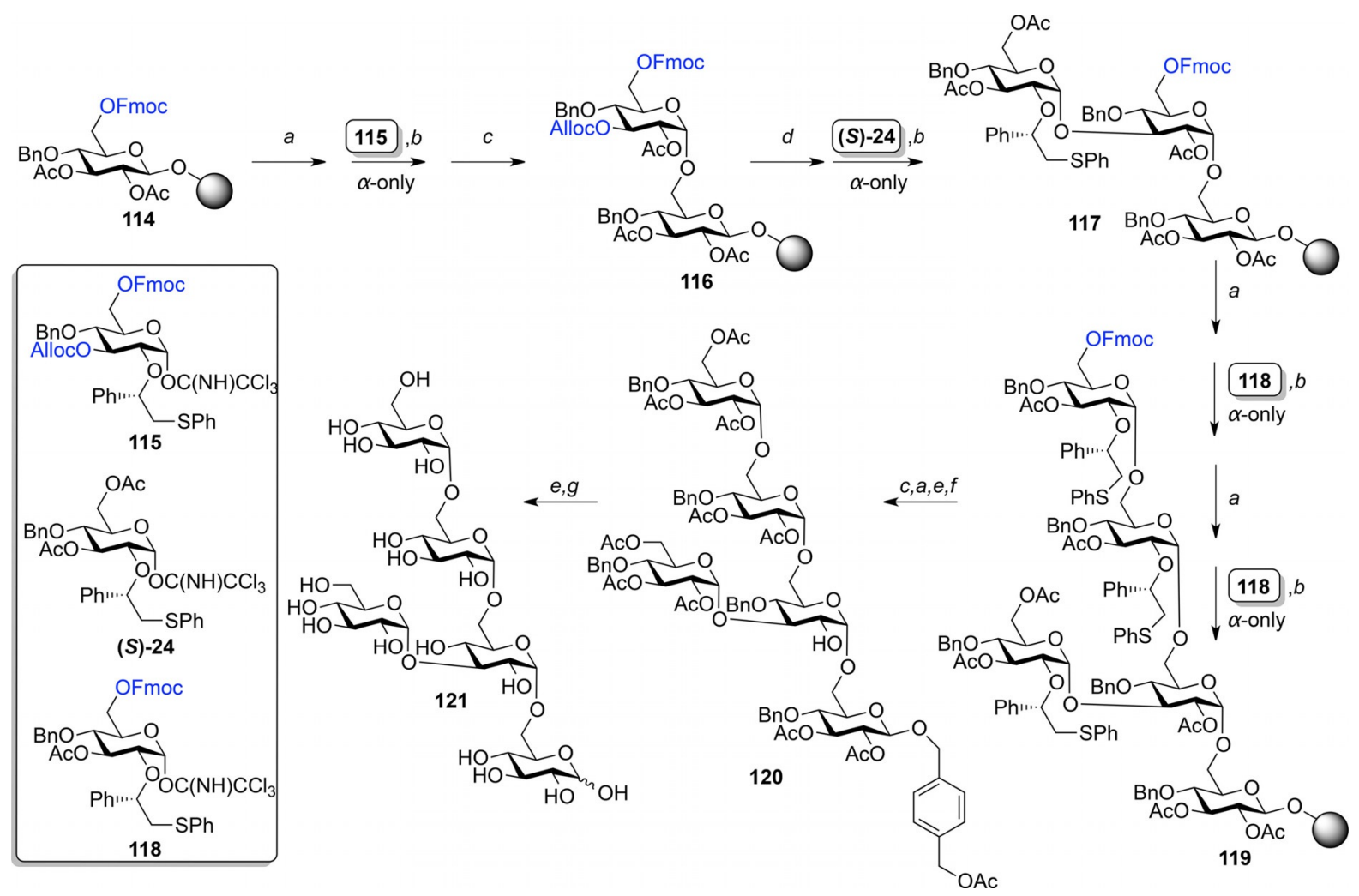

Scheme 10. Solid phase synthesis of branched pentaglucoside 121. Reagents and conditions: a) $10 \%$ v/v piperidine in DMF, 5 min, RT. b) 115 , (S)-24 or 118,

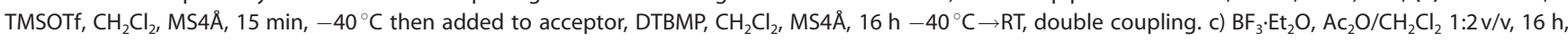
$0{ }^{\circ} \mathrm{C}$. d) $40 \mathrm{~mol} \% \mathrm{Pd}\left(\mathrm{PPh}_{3}\right)_{4}, \mathrm{THF} / \mathrm{AcOH}$ 10:1 v/v, 16 h, RT. e) $\mathrm{NaOMe}, \mathrm{MeOH} / \mathrm{CH}_{2} \mathrm{Cl}_{2}$ 1/1, 16 h, RT. f) $\left.\mathrm{Ac}_{2} \mathrm{O}, \mathrm{pyr} 1: 3 \mathrm{v} / \mathrm{v} ; \mathrm{g}\right) 20 \mathrm{wt} \% \mathrm{Pd}(\mathrm{OH})_{2} / \mathrm{C}, \mathrm{H}_{2}, \mathrm{EtOH} / \mathrm{H}_{2} \mathrm{O} 1: 1 \mathrm{v} /$ v, 16 h, RT, $87 \%$. 


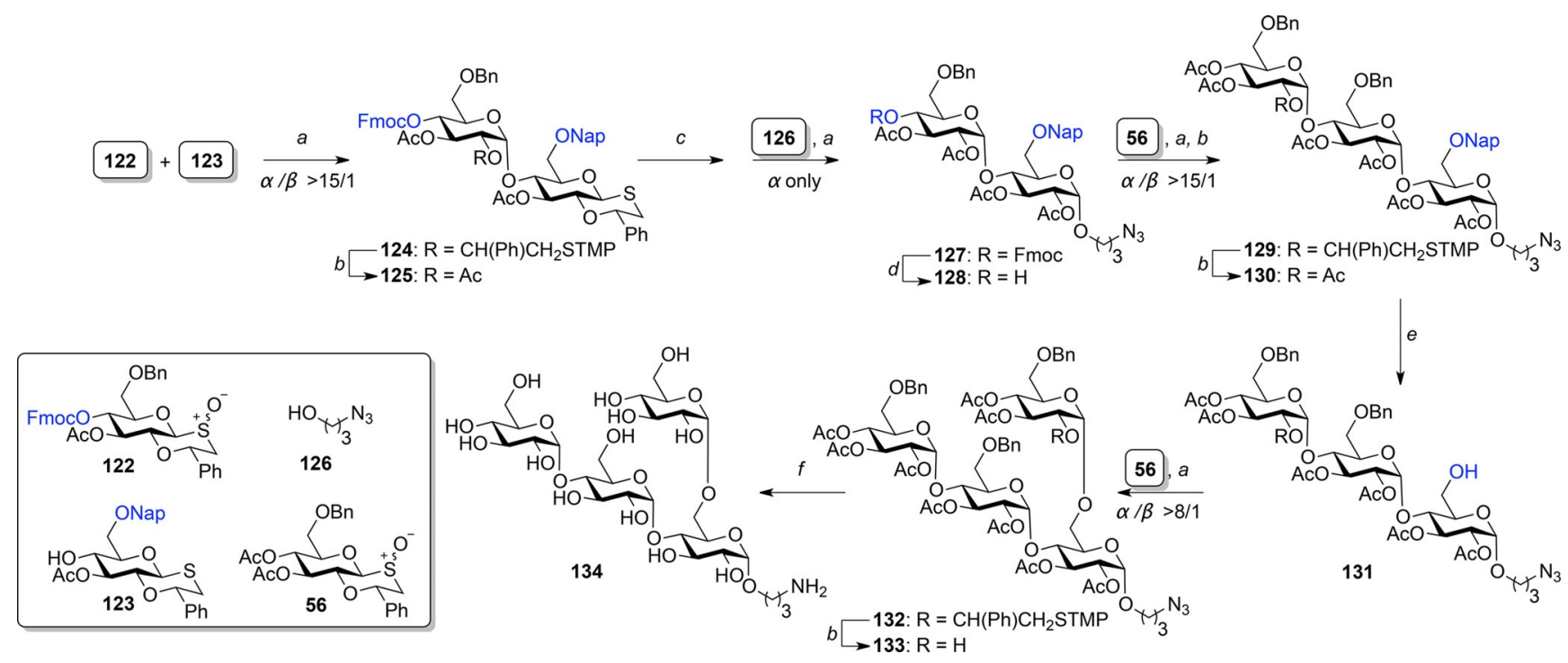

Scheme 11. Synthesis of branched tetraglucoside 134. Reagents and conditions: a) 1,3,5-trimethoxybenzene, Tf ${ }_{2} \mathrm{O}, \mathrm{DTBMP}$, then $123,126,128$ or $\mathbf{1 3 1}$; b) $10 \%$ TFA, DCM, then $\mathrm{Ac}_{2} \mathrm{O}$, Pyr. c) $m$-CPBA, DCM d) $20 \% \mathrm{NMP}$ in DMF e) DDQ, $\mathrm{H}_{2} \mathrm{O}, \mathrm{DCM}$ f) $\mathrm{NaOMe}, \mathrm{MeOH}, \mathrm{then} \mathrm{H}_{2}, \mathrm{Pd}(\mathrm{OH})_{2} / \mathrm{C}, \mathrm{tBuOH}, \mathrm{H}_{2} \mathrm{O}, \mathrm{AcOH}$. (NMP $=\mathrm{N}-$ methyl-2-pyrrolidone).

Similar $\alpha$-glucans have also been isolated from various microbial sources such as Pseudallescheria boydii, producing a polysaccharide that is essential for conidial phagocytosis by macrophages and induction of innate immune responses in a TLR2-dependent manner. Tetrasaccharide 134 isolated from Pseudallescheria boydii was synthesized using oxathiane donors and a bi-directional approach. ${ }^{[28]}$ Tetrasaccharide 134 contains an $\alpha(1,4)$-linked backbone and $\alpha(1,6)$-branching which could be prepared from monosaccharides building blocks 56, 122, 123 and 126 (Scheme 11). It was envisioned that oxathiane 123 could serve as a latent-active glycosyl donor. ${ }^{[4]}$ Hence, disaccharide 124 was prepared by coupling of preactivated 122 to glycosyl acceptor 123 with excellent selectivity. Activation of this disaccharide required oxidation to the sulfoxide and hence the C-2 auxiliary was removed to prevent oxidation at this site using $10 \%$ trifluoroacetic acid (TFA) in DCM, followed by acetylation (125). Next, oxidation of the oxathiane with $m$ CPBA-enabled sulfonium ion synthesis and coupling with amino spacer 126 with excellent selectivity. Fmoc removal allowed for $\alpha(1,4)$-extention at the non-reducing end using preactivated 56 to afford trisaccharide 129 in good yield and excellent selectivity. Again, the auxiliary was removed prior to further extension to afford 130. Installation of the $\alpha(1,6)$ branch was achieved after removal of the 2-methylnaphthyl ether and coupling with preactivated 56 . The $\alpha$-selectivity was moderate $(8: 1, \alpha / \beta)$, but could be improved by the use of a different oxathiane sulfoxide donor. Final deprotection afforded tetrasaccharide 134 . The use of oxathiane donors is very attractive since the oxathiane is stable during protecting group manipulations, can serve as a glycosyl acceptor and can be activated by oxidation to the corresponding sulfoxide which can be used as a glycosyl donor. A major drawback is that prior to oxidation of the oxathiane, the removal of $\mathrm{C}-2$ auxiliaries is required to prevent oxidation at this position. Also, the oxathiane donors used in this study are less selective than the second generation auxiliaries used on a solid support.
Both the oxathiane donor and donor bearing a C-2 (S)-phenylthiomethylbenzyl ether were employed for the synthesis of oligosaccharide 149 found on the glycosylphosphatidylinositol (GPI) anchor of Trypanosoma brucei, a parasite that causes sleeping sickness in humans and similar diseases in domestic animals (Scheme 12). ${ }^{[42 b, 47]}$ Hexasaccharide 149 from T. brucei consists of four galactose and two mannose monomers all linked by $\alpha$-glycosidic bonds. The synthesis was carried out using a light fluorous tag (F) at the reducing end. This tag allows glycosylation to take place in solution, but facilitates purification by fluorous solid-phase extraction (F-SPE) obviating the need for chromatography and is even amendable to automation. ${ }^{[48]}$ The synthesis was rehearsed and optimized using traditional solution phase chemistry and the optimized procedure was next used to enable rapid synthesis using F-SPE.

Each glycosylation was performed twice, in order to ensure complete conversion of the glycosyl acceptor. The synthesis started with the addition of preactivated oxathiane donor 136, to $\alpha$-glucoside 135 bearing the fluorous tag, forming the disaccharide as the $\alpha(1,3)$-linkage only. Removal of the auxiliary with $10 \%$ TFA in DCM afforded acceptor 137. Addition of preactivated oxathiane donor 141 to 137 did not result in product formation and using an excess of donor 141 resulted in a disappointing $25 \%$ yield of the corresponding $\alpha-1,2$-linked trisaccharide. This problem was overcome by implementation of the more reactive C-2 benzyl protected donor 138, activated by triflic acid, forming of trisaccharide 139 with $\alpha / \beta$ 15:1. In order to avoid overoxidation, careful oxidative deprotection of the Nap ether was achieved using a small excess of 2,3-dichloro5,6-dicyano-1,4-benzoquinone (DDQ) in $\mathrm{H}_{2} \mathrm{O}$, affording alcohol 140. Addition of preactivated donor 141 and subsequent acidic deprotection of the C-2 auxiliary afforded tetrasaccharide 142 as only the $\alpha$-anomer. Formation of pentasaccharide 144 by employing preactivated oxathiane donor 141, surprisingly resulted in a disappointing yield of $20 \%$. Addition of tetrasaccharide 142 to preactivated 143 , followed by acidic re- 

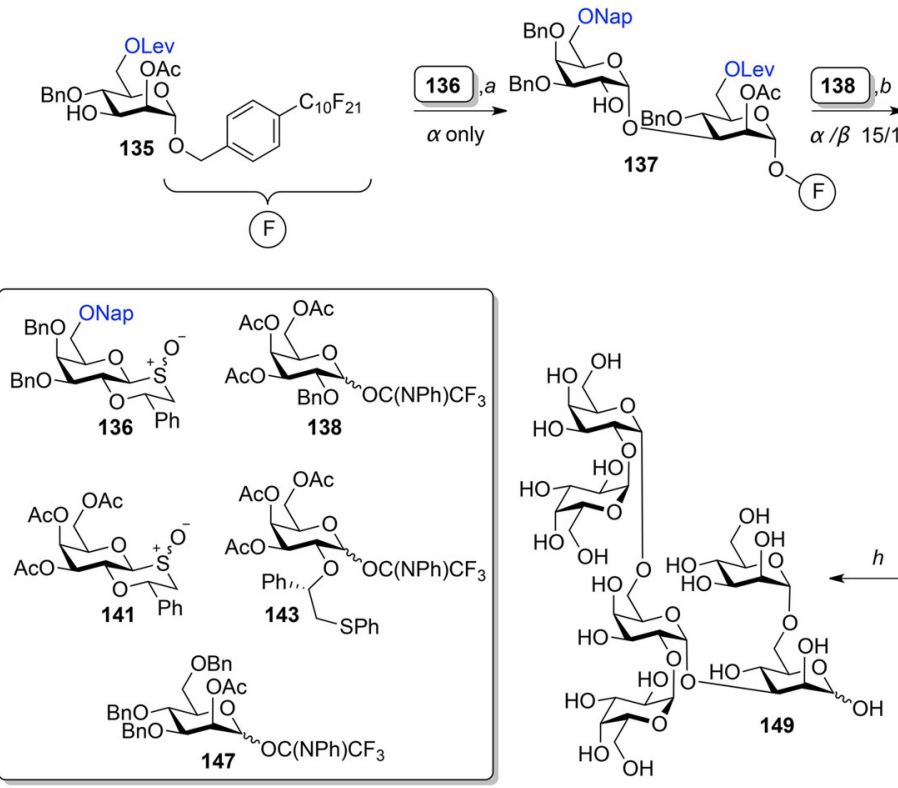

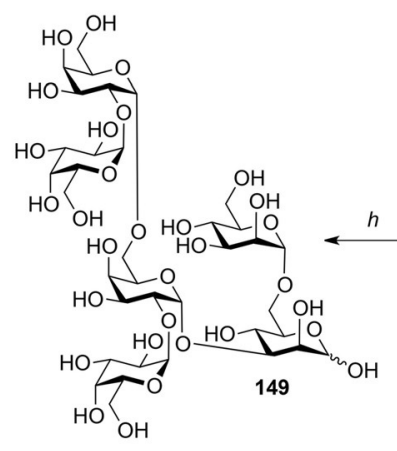

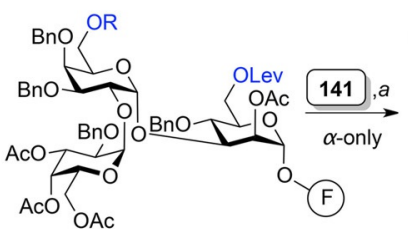

$c \longrightarrow$ 139: $R=\mathrm{Nap}$
$\longrightarrow 140: \mathrm{R}=\mathrm{H}$

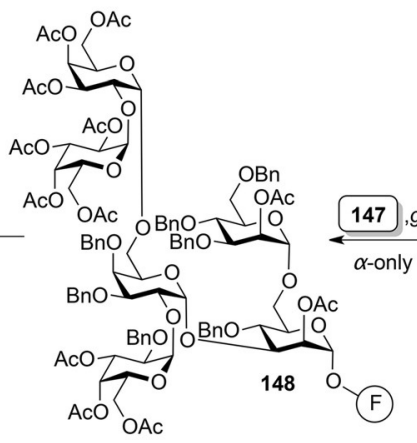

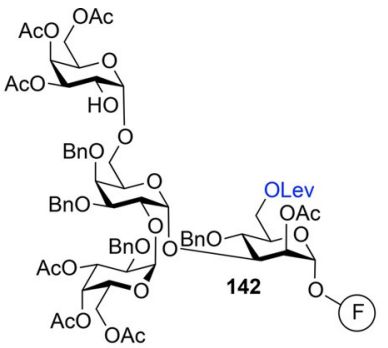

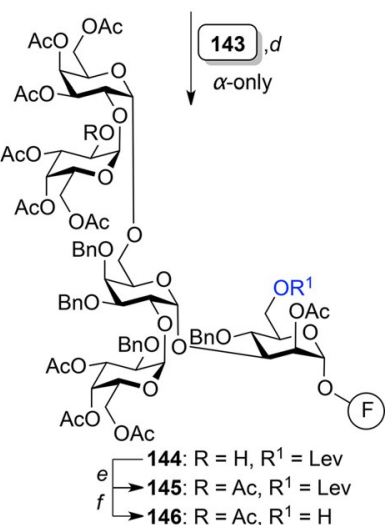

Scheme 12. Synthesis of $T$. brucei GPI oligosaccharide 149. Reagents and conditions: a) 1,3,5-trimethoxybenzene, Tf ${ }_{2} \mathrm{O}$, DTBMP, then 135 or 140 . then $10 \%$ TFA, DCM; b) TfOH, DCM; c) DDQ, $\mathrm{H}_{2} \mathrm{O}, \mathrm{DCM}$; d) TfOH, then 142, DTBMP, then $10 \% \mathrm{TFA}$, DCM; e) Ac $\mathrm{O}_{2}$, Pyr., DMAP; f) NH $\mathrm{NH}_{2} \cdot \mathrm{AcOH}$, pyr.; g) TMSOTf, 146 , $\mathrm{DCM}$; h) $\mathrm{H}_{2}, \mathrm{Pd} / \mathrm{C}, \mathrm{MeOH}, \mathrm{AcOH}$, then $\mathrm{NaOMe}, \mathrm{MeOH}$. (DMAP =4-dimethylaminopyridine).

moval of the auxiliary resulted in pentasaccharide 144 as only the $\alpha$-anomer in $76 \%$ yield. Acetylation and subsequent removal of the levulinoyl (Lev)-protected ester using hydrazinium acetate yielded acceptor 146. As discussed earlier, acyl participation in manno-type glycosylations reliably affords $\alpha$-linked products and thus TMSOTf mediated glycosylation of mannoside 147 with acceptor 146 cleanly afforded hexasaccharide 148. Hydrogenation of the benzyl ethers and subsequent deacetylation afforded target hexasaccharide 149 . The overall yield of the solution phase synthesis was $9 \%$, whereas the fluorous-assisted solid phase synthesis afforded hexasaccharide 149 in $17 \%$ overall yield corresponding to an impressive $85 \%$ yield per reaction step. Additionally, the authors needed only six days to complete the synthesis, which can probably be sped up further by using automated fluorous-supported synthesis. The synthesis of this hexasaccharide highlights the progress made in the stereoselective synthesis of $\alpha$-glycosides, but also shows one of its weaknesses. Combination of the bulky C-2 sulfonium intermediate with an unreactive acceptor (i.e. alcohol on C-2), affords poor to no glycosylation.

\section{Summary and Outlook}

The use of C-2 chiral auxiliaries for the stereoselective synthesis of 1,2-cis-glycosides holds considerable potential to become a general method to prepare this class of molecules. In most cases neighboring group participation of the C-2 auxiliary during glycosylation leads to the formation of a trans-decalin intermediate that can be displaced to form 1,2-cis-glycosides. Whether the observed trans-decalin intermediate is a reactive species or merely a resting state seems to be strongly dependent on the structure of the auxiliary and the protecting groups on the glycosyl donor (Scheme 13). In general, electron withdrawing protecting groups perform better than electron donating protecting groups yet more work is needed to establish the exact mechanism of sulfonium ion displacement. The structure of the C-2 auxiliary is another major factor that determines the stereoselectivity and auxiliaries carrying a chiral group that promote the formation of the trans-decalin intermediate versus the cis-decalin are markedly more stereoselective than the achiral variants. Furthermore, auxiliaries containing a thioether as the nucleophilic group to produce sulfonium ion intermediates perform better than variants containing an ester, ether, selenoether, or iodo group. Reaction parameters such as temperature, solvent, acceptor type etc. are also important, yet the influence of such parameters are often difficult to establish since researchers use different glycosylation proto-

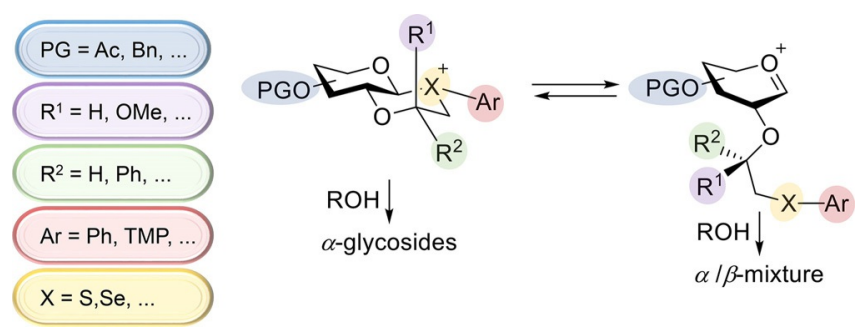

Scheme 13. Factors that influence the reactivity of sulfonium ions in glycosylations. 
cols. It would therefore be desirable to standardize the protocol to make studies by different researchers comparable.

The use of chiral auxiliaries is generally applicable to glucose, galactose and mannose, but further research is needed to expand the scope to their aminoglycoside derivatives (glucosamine, galactosamine, mannosamine etc.) and other monosaccharides (fucose, xylose, rhamnose, etc.). Finally, the use of chiral auxiliaries to prepare complex oligosaccharides has been demonstrated in solution as well as on solid phase. Excellent 1,2-cis-selectivity was obtained in most cases, but especially sterically hindered glycosyl acceptors proved difficult to glycosylate. In these cases, more conventional glycosyl donors carrying a non-participation functionality proved to be more efficient. In conclusion, chiral auxiliaries to prepare 1,2-cis-glycosides is a promising technology which, combined with the well-established use of C-2 acyl participation to prepare 1,2trans-glycosides, will provide access to the major linkage types found in natural oligosaccharides.

\section{Acknowledgements}

This review was prepared with the support of an NWO-VENI grant awarded to T.J.B.

\section{Conflict of interest}

The authors declare no conflict of interest.

Keywords: chiral auxiliary · glycosylation · oligosaccharides stereoselectivity $\cdot$ sulfonium ion

[1] A. Varki, Glycobiology 1993, 3, 97-130.

[2] J. D. Marth, Nat. Cell Biol. 2008, 10, 1015-1016

[3] G. W. Hart, R. J. Copeland, Cell 2010, 143, 672-676.

[4] T. J. Boltje, T. Buskas, G.-J. Boons, Nat. Chem. 2009, 1, 611-622.

[5] X. Zhu, R. R. Schmidt, Angew. Chem. Int. Ed. 2009, 48, 1900-1934; Angew. Chem. 2009, 121, 1932-1967.

[6] S. S. Nigudkar, A. V. Demchenko, Chem. Sci. 2015, 6, 2687-2704.

[7] H. Paulsen, Angew. Chem. Int. Ed. Engl. 1982, 21, 155-173; Angew. Chem. 1982, 94, 184-201.

[8] M. H. S. Marqvorsen, M. J. Pedersen, M. R. Rasmussen, S. K. Kristensen, R. Dahl-Lassen, H. H. Jensen, J. Org. Chem. 2017, 82, 143-156.

[9] R. Enugala, L. C. R. Carvalho, M. J. Dias Pires, M. M. B. Marques, Chem. Asian J. 2012, 7, 2482-2501.

[10] a) D. Crich, Acc. Chem. Res. 2010, 43, 1144-1153; b) H. Elferink, R. A. Mensink, P. B. White, T. J. Boltje, Angew. Chem. Int. Ed. 2016, 55, 1121711220; Angew. Chem. 2016, 128, 11383-11386.

[11] a) E. A. Mensah, F. Yu, H. M. Nguyen, J. Am. Chem. Soc. 2010, 132, 14288-14302; b) J. D. M. Olsson, L. Eriksson, M. Lahmann, S. Oscarson, J. Org. Chem. 2008, 73, 7181-7188.

[12] A. Imamura, H. Ando, S. Korogi, G. Tanabe, O. Muraoka, H. Ishida, M. Kiso, Tetrahedron Lett. 2003, 44, 6725-6728.

[13] X. Zhu, S. Kawatkar, Y. Rao, G.-J. Boons, J. Am. Chem. Soc. 2006, 128, $11948-11957$.

[14] S.-R. Lu, Y.-H. Lai, J.-H. Chen, C.-Y. Liu, K.-K. T. Mong, Angew. Chem. Int. Ed. 2011, 50, 7315-7320; Angew. Chem. 2011, 123, 7453-7458.

[15] a) F. Barresi, O. Hindsgaul, J. Am. Chem. Soc. 1991, 113, 9376-9377; b) K.-H. Jung, M. Müller, R. R. Schmidt, Chem. Rev. 2000, 100, $4423-$ 4442; c) A. Ishiwata, Y. J. Lee, Y. Ito, Org. Biomol. Chem. 2010, 8, $3596-$ 3608.
[16] M. T. C. Walvoort, J. Dinkelaar, L. J. van den Bos, G. Lodder, H. S. Overkleeft, J. D. C. Codée, G. A. van der Marel, Carbohydr. Res. 2010, 345, $1252-1263$.

[17] S. Manabe, K. Ishii, Y. Ito, J. Am. Chem. Soc. 2006, 128, 10666-10667.

[18] J.-H. Kim, H. Yang, G.-J. Boons, Angew. Chem. Int. Ed. 2005, 44, 947-949; Angew. Chem. 2005, 117, 969-971.

[19] J. Xue, Z. Guo, Tetrahedron Lett. 2001, 42, 6487-6489.

[20] J.-H. Kim, H. Yang, V. Khot, D. Whitfield, G.-J. Boons, Eur. J. Org. Chem. 2006, 5007-5028.

[21] J.-H. Kim, H. Yang, J. Park, G.-J. Boons, J. Am. Chem. Soc. 2005, 127, $12090-12097$.

[22] D. J. Fox, D. House, S. Warren, Angew. Chem. Int. Ed. 2002, 41, 2462 2482; Angew. Chem. 2002, 114, 2572-2593.

[23] T. J. Boltje, J.-H. Kim, J. Park, G.-J. Boons, Org. Lett. 2011, 13, 284-287.

[24] L. Bohé, D. Crich, Carbohydr. Res. 2015, 403, 48-59.

[25] T. Fang, Y. Gu, W. Huang, G.-J. Boons, J. Am. Chem. Soc. 2016, 138, $3002-3011$.

[26] J. Park, T. J. Boltje, G.-J. Boons, Org. Lett. 2008, 10, 4367-4370.

[27] M. A. Fascione, S. J. Adshead, S. A. Stalford, C. A. Kilner, A. G. Leach, W. B. Turnbull, Chem. Commun. 2009, 5841-5843.

[28] T. Fang, K.-F. Mo, G.-J. Boons, J. Am. Chem. Soc. 2012, 134, 7545-7552.

[29] M. A. Fascione, C. A. Kilner, A. G. Leach, W. B. Turnbull, Chem. Eur. J. 2012, 18, $321-333$

[30] M. A. Fascione, W. B. Turnbull, Beilstein J. Org. Chem. 2010, 6, 19.

[31] E. Schaumann, S. Scheiblich, Tetrahedron Lett. 1985, 26, 5269-5272.

[32] M. A. Fascione, N. J. Webb, C. A. Kilner, S. L. Warriner, W. B. Turnbull, Carbohydr. Res. 2012, 348, 6-13.

[33] a) D. J. Cox, A. J. Fairbanks, Tetrahedron: Asymmetry 2009, 20, 773-780; b) A. J. A. Watson, S. R. Alexander, D. J. Cox, A. J. Fairbanks, Eur. J. Org. Chem. 2016, 1520-1532.

[34] D. J. Cox, G. P. Singh, A. J. A. Watson, A. J. Fairbanks, Eur. J. Org. Chem. 2014, 4624-4642.

[35] G. P. Singh, A. J. A. Watson, A. J. Fairbanks, Org. Lett. 2015, 17, 4376 4379.

[36] R. A. Mensink, H. Elferink, P. B. White, N. Pers, F. P. J. T. Rutjes, T. J. Boltje, Eur. J. Org. Chem. 2016, 4656-4667.

[37] a) R. M. Beesley, C. K. Ingold, J. F. Thorpe, J. Chem. Soc. Trans. 1915, 107, $1080-1106$; b) S. M. Bachrach, J. Org. Chem. 2008, 73, 2466-2468.

[38] M.-Z. Liu, H.-N. Fan, Z.-W. Guo, Y.-Z. Hui, Chin. J. Chem. 1996, 14, $190-$ 192.

[39] A. V. Demchenko, E. Rousson, G.-J. Boons, Tetrahedron Lett. 1999, 40, $6523-6526$.

[40] K. S. Kim, J. H. Kim, Y. J. Lee, Y. J. Lee, J. Park, J. Am. Chem. Soc. 2001, 123, $8477-8481$.

[41] a) L. J. van den Bos, J. D. C. Codée, J. C. van der Toorn, T. J. Boltje, J. H. van Boom, H. S. Overkleeft, G. A. van der Marel, Org. Lett. 2004, 6, $2165-2168$; b) L. J. van den Bos, R. E. J. N. Litjens, R. J. B. H. N. van den Berg, H. S. Overkleeft, G. A. van der Marel, Org. Lett. 2005, 7, 2007 2010.

[42] a) T. J. Boltje, J.-H. Kim, J. Park, G.-J. Boons, Nat. Chem. 2010, 2, 552 557; b) W. Huang, Q. Gao, G.-J. Boons, Chem. Eur. J. 2015, 21, 1292012926.

[43] P. H. Seeberger, Chem. Soc. Rev. 2008, 37, 19-28.

[44] a) S. Jonke, K.-g. Liu, R. R. Schmidt, Chem. Eur. J. 2006, 12, 1274-1290; b) D. B. Werz, B. Castagner, P. H. Seeberger, J. Am. Chem. Soc. 2007, 129, 2770-2771; c) J.D. C. Codée, L. Kröck, B. Castagner, P. H. Seeberger, Chem. Eur. J. 2008, 14, 3987-3994.

[45] C. Zhao, M. Li, Y. Luo, W. Wu, Carbohydr. Res. 2006, 341, 485-491.

[46] a) S. Mehta, B. Mario Pinto, Tetrahedron Lett. 1991, 32, 4435-4438; b) R. Roy, F. O. Andersson, M. Letellier, Tetrahedron Lett. 1992, 33, 60536056 ; c) G.-J. Boons, B. Heskamp, F. Hout, Angew. Chem. Int. Ed. Engl. 1996, 35, 2845-2847; Angew. Chem. 1996, 108, 3053-3056.

[47] M. Ferguson, S. Homans, R. Dwek, T. Rademacher, Science 1988, 239, $753-759$.

[48] a) W. Zhang, D. P. Curran, Tetrahedron 2006, 62, 11837-11865; b) S.-L. Tang, N. L. B. Pohl, Org. Lett. 2015, 17, 2642-2645.

Manuscript received: February 27, 2017

Accepted manuscript online: July 25, 2017

Version of record online: October 4, 2017 